\title{
Statistical Mechanics of Red Blood Cell Aggregation: The Distribution of Rouleaux in Thermal Equilibrium
}

\author{
Frederik W. Wiegel ${ }^{1}$ and Alan S. Perelson ${ }^{2}$ \\ Received February 11, 1982; revised July 26, 1982
}

\begin{abstract}
When placed in suspension red blood cells adhere face-to-face and form long, cylindrical, and sometimes branched structures called rouleaux. We use methods developed in statistical mechanics to compute various statistical properties describing the size and shape of rouleaux in thermodynamic equilibrium. This leads to analytical expressions for (1) the average number of rouleaux consisting of $n$ cells and having $m$ branch points; (2) the average number of cells per rouleau; (3) the average number of branch points per rouleau; and (4) the number of rouleaux with $n$ cells in a system containing a total of $N$ cells. We also derive asymptotic formulas that simplify these analytic expressions, and present numerical comparisons of the exact and asymptotic results.
\end{abstract}

KEY WORDS: Aggregation; red blood cells; rouleaux; statistical mechanics; rooted trees.

\section{INTRODUCTION}

When blood flow is stopped or substantially slowed the red blood cells, which had formed a monodisperse suspension, tend to aggregate into long, cylindrical, and sometimes branched objects called rouleaux. The cells within a rouleau generally adhere face-to-face and the rouleau resembles stacks of coins joined together into a treelike structure. Figure 1 illustrates the geometrical form of some typical rouleaux.

\footnotetext{
${ }^{1}$ Department of Applied Physics, Twente University of Technology, P.O. Box 217, Enschede, The Netherlands.

${ }^{2}$ Theoretical Division, University of California, Los Alamos National Laboratory, Los Alamos, New Mexico 87545.
} 

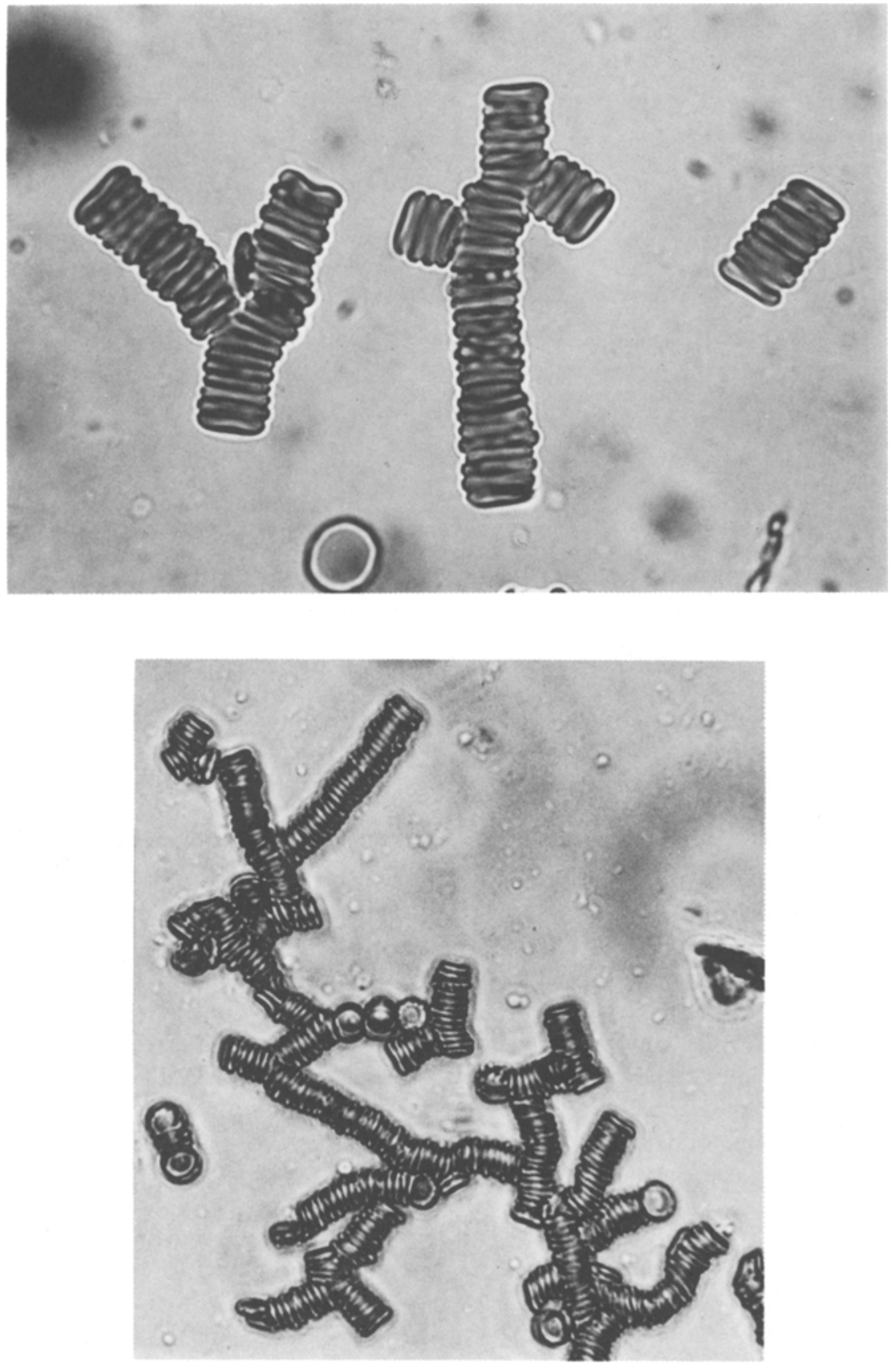

Fig. 1. Rouleaux that formed spontaneously from normal human blood. (a) Typical small rouleaux. (b) A larger rouleau. 
Rouleau formation should be of general interest to both experimentalists and theorists as a model system for the study of cellular adhesion and aggregation under physiological conditions. Erythrocyte aggregation is also of clinical interest since the appearance of large rouleaux increases the viscosity and the sedimentation rate of blood. The erythrocyte sedimentation rate is measured diagnostically and indicates the presence of elevated blood plasma concentrations of certain macromolecules, such as immunoglobulin and fibrinogen, characteristic of various disease states. ${ }^{(1,2)}$ The increase in viscosity accompanying rouleau formation affects the hemodynamics of the microcirculation and has been implicated as the cause of complications seen in a number of diseases. ${ }^{(3)}$

The size and shape of rouleaux are important in determining their physiological effects. In thermal equilibrium the size distribution of rouleaux and certain statistical properties characteristic of rouleau shape can be calculated using the techniques of statistical mechanics. Demonstrating how such calculations can be done forms the subject of this paper (biophysical applications have been published elsewhere ${ }^{(4)}$ ).

Under physiological conditions rouleau formation is a time-dependent phenomenon. The process of rouleau formation has recently been modeled by Samsel and Perelson ${ }^{(5,6)}$ using sets of coupled kinetic equations. The equilibrium distribution to be derived in this paper should correspond to the infinite time limit of the theory of Samsel and Perelson. ${ }^{(6)}$

In the analysis presented here we only consider red cell aggregation in the absence of fluid flow. Hydrodynamic aspects of rouleau formation as studied, for example, by Goldsmith and Mason ${ }^{(7,10)}$ or Adler $^{(11)}$ will not be dealt with. One of the main motivations for our work is that the influence of hydrodynamic effects on the rouleau size distribution can most easily be ascertained by neglecting these effects altogether and comparing the predicted size distribution with the observed one. The problem of calculating the rouleau size distribution in a shear flow, which when large rouleau form requires a combination of the mechanics of fluids in porous media ${ }^{(12)}$ and statistical considerations, will be left aside for the time being.

In this paper we shall consider the following problem. A large number of red blood cells, $N$, are placed in a fluid at rest in a volume $V$, at an absolute temperature $T$ and form rouleaux. We assume that two energetically distinct adhesion processes occur as shown in Fig. 2: (a) two cells adhere face-to-face with a binding energy $-E_{A}<0$; (b) three cells adhere forming a branch point, with binding energy $-E_{B}<0$. As a result of these adhesion processes, the red cells form treelike structures. The process of tree formation is counteracted by random thermal motion (Brownian movement) which tends to break up rouleaux, especially large ones which can disintegrate in many different ways. Our goal is to calculate the 


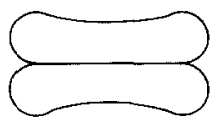

(o)

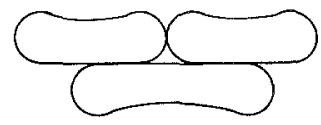

(b)

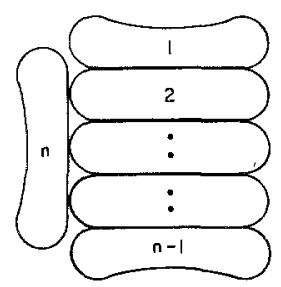

(c)

Fig. 2. (a) Two cells adhere with energy $-E_{A}<0$. (b) Three cells adhere forming a branch point with energy $-E_{B}<0$. (c) A branch point formed by $n$ cells.

resulting equilibrium distribution of rouleaux, i.e., the number of rouleaux of a given size after an infinitely long time. We shall also calculate the equilibrium distribution of the number of branch points in rouleaux of a given size. Before proceeding with the theory some comments are appropriate.

Our model involves two basic energetic processes, chain elongation and chain branching. In our statistical mechanical calculations we shall generally not use the energies $E_{A}$ and $E_{B}$ themselves, but rather the "Boltzmann factors"

$$
\alpha^{\prime}=\exp \left(E_{A} / k_{B} T\right) \text { and } \beta^{\prime}=\exp \left(E_{B} / k_{B} T\right)
$$

where $k_{B}$ is Boltzmann's constant. Each red cell has two faces. Thus when a cell joins to an existing chain and elongates it, the adhesion can be to either of two faces. The variable

$$
\alpha=2 \alpha^{\prime}
$$

will be used to denote the Boltzmann factor for elongation multiplied by the number of ways the interaction can occur. Similarly, when two cells adhere to a cell at the end of a chain to form a branch point, each cell can adhere with either of two faces. The variable

$$
\beta=4 \beta^{\prime}
$$

will be used to denote the Boltzmann factor for branching multiplied by the number of ways the interaction can occur.

The values of $E_{A}$ and $E_{B}$ depend on the type and concentration of the macromolecules that form the intercellular bridges mediating cellular adhe- 
sion, the ionic composition of the medium, the surface charge on the erythrocytes, and the viscoelastic properties of the red cell membrane. We expect

$$
E_{A} \leqslant E_{B} \leqslant 2 E_{A}
$$

If $E_{A}$ were greater than $E_{B}$ then almost no branching would be present at equilibrium, contrary to observation. The three cells forming a branch point can at best adhere over an area twice that of two cells (see Fig. 2) and consequently we expect $E_{B} \leqslant 2 E_{A}$. For stable adhesion both $E_{A}$ and $E_{B}$ will be large compared to the thermal energy $k_{B} T$, and hence $\alpha^{\prime}$ and $\beta^{\prime}$ will be large compared to unity.

Although we assume that three cells form a branch point our methods can easily be generalized to include the case in which a larger number of cells are involved as illustrated in Fig. 2c. If a single cell adheres to $n-1$ other cells, creating a branch point involving $n$ cells, then

$$
E_{B}=(n-2) E_{A}+E_{n-1}
$$

where $E_{n-1}$ is the energy characterizing the binding of one cell to the edges of $n-1$ other cells. In a stack of $n-1$ cells there are $n-2$ interfaces, each contributing an energy $E_{A}$. Furthermore, for such multicell branch points

$$
\beta=2^{n-1} \beta^{\prime}
$$

The assumption that rouleaux are treelike structures plays a central role in our analysis. At very large rouleau sizes this assumption may break down as a result of the formation of cycles in which a branch of a tree will attach itself to some other branch of the same tree. The phenomenon of cycle formation leads to tremendous mathematical complications which have not yet been resolved. There is some hope, however, that the method of the renormalization group can be used to gain insight into some of these problems. ${ }^{(13-15)}$

In order to characterize the rouleaux distribution at equilibrium we introduce the following quantities: (a) The average number of rouleaux that consist of $n$ cells and have $m$ branch points, $R_{n, m}^{*}$. This quantity leads, among others, to the degree of branching of the "average" rouleau and should, therefore, play a role in determining the sedimentation rate of a rouleau. (b) The average number of rouleaux consisting of $n$ cells, $R_{n}^{*}$. (c) The average number of cells per rouleau, $\langle n\rangle$. (d) The average number of branch points per rouleau, $\langle m\rangle$.

\section{GENERAL CONSIDERATIONS}

We apply the general scheme of equilibrium statistical mechanics to the computation of the rouleau distribution. Although the methods we 
employ are discussed in many texts (cf. Refs. 16-18), we shall briefly explain and motivate the methods so as to make our treatment intelligible to a biophysical audience. We begin with a number of definitions.

A macrostate of the system is a collection of numbers $\left\{R_{n}\right\} \equiv\left(R_{1}, R_{2}\right.$, $R_{3}, \ldots$ ), where $R_{n}$ denotes the number of rouleaux consisting of exactly $n$ cells. The macrostate can be observed experimentally. A microstate of the system is a particular assignment of the $N$ red blood cells to the various rouleaux, including their ordering within each rouleau, and an assignment of the positions of the various rouleaux within the volume $V$. Microstates are characteristic of the state of each individual red cell and generally are not observed experimentally.

To utilize the methods of statistical mechanics we first calculate the configuration sum $Q\left\{\boldsymbol{R}_{n}\right\}$, defined by

$$
Q\left\{R_{n}\right\}=\sum_{C}^{\prime} \exp \left[-E(C) / k_{B} T\right]
$$

where the prime indicates a summation over all those microstates that are compatible with the macrostate $\left\{R_{n}\right\}$ and where $E(C)$ denotes the energy of microstate $C$. If there is a single macrostate $\left\{R_{n}^{*}\right\}$ obtained from the overwhelming majority of all possible microstates, then that state is the one that would be observed. Thus the most probable distribution $\left\{R_{n}^{*}\right\}$ would correspond to the equilibrium rouleau distribution. The most probable distribution can be calculated by the standard technique of maximizing $Q\left\{R_{n}\right\}$ with respect to variations of the $R_{n}$ that are compatible with the constraint that the total number of cells is constant, i.e.,

$$
\sum_{n=1}^{\infty} n R_{n}=N
$$

The assumption that the equilibrium distribution is the most probable distribution is only valid when the number of states is so large that fluctuations around the most probable distribution are neglible (cf. Ref. 19). One can rigorously show that this is the case in the "thermodynamic limit," i.e., when $N \rightarrow \infty, V \rightarrow \infty$, but the cell density $N / V$ remains finite. Here we shall assume that this is true. Thus our method will only be valid for studies employing a large volume of blood containing a large number of erythrocytes, as is usually the case. Donoghue and Gibbs ${ }^{(20)}$ and Donoghue ${ }^{(21)}$ have recently developed methods which can be used to rigorously calculate the mean rouleau distribution in a system with a finite number of red cells.

The calculation of $Q\left\{R_{n}\right\}$ proceeds in several steps. First we count the number of microstates consistent with a given macrostate. To do this we note that there are many ways to divide $N$ red blood cells into groups, each 
of which corresponds to one rouleau, such that there are exactly $R_{n}$ groups of $n$ cells each, $n=1,2, \ldots$. Call the number of ways this division can be made $\Omega\left\{R_{n}\right\}$; it can be calculated as follows. Consider one particular partition of the $N$ cells into $R_{n}$ groups of $n$ cells each. Put the $N$ cells in a linear sequence, beginning with the cells in the $R_{1}$ rouleaux of 1 cell, followed by the cells in the $R_{2}$ rouleaux of 2 cells, etc. This gives a permutation of the $N$ cells. There are $N$ ! permutations of $N$ cells. However, all permutations do not lead to distinct microstates. This is the case because, first, the $n$ cells in each group of $n$ can be incorporated in the linear sequence in $n$ ! different ways and, second, the $R_{n}$ groups of $n$ cells each can be put down in the sequence in $R_{n}$ ! ways. Hence one has

$$
\Omega\left\{R_{n}\right\}=\frac{N !}{\prod_{n=1}^{\infty}\left(R_{n} ! n !^{R_{n}}\right)}
$$

In the second step in the calculation of $Q\left\{R_{n}\right\}$ we consider one of these $\Omega$ different partitions. Take one of its rouleaux; this rouleau can be moved around through the volume $V$. This leads roughly to

$$
N_{0} \equiv V / v
$$

different microstates per rouleau. Here $v$ is some elementary volume of the order of the volume of a red blood cell. Thus we are taking the view that we can specify the location of a rouleau by specifying the position of a single cell within the rouleau. In Section 3 we will consider the additional microstates that occur if rotations about the center of mass of the rouleau are also counted.

Having now fixed this specific rouleau in some position in space, its microstates can be arranged in groups of $n$ ! states which arise out of each other by permuting the $n$ cells in the rouleau among each other. Hence the sum of the Boltzmann factors over all the microstates of this particular rouleau can be written in the form $N_{0} n ! Q_{n}$, where $Q_{n}$ denotes the sum of the Boltzmann factors of all those microstates in which the rouleaux (a) consists of $n$ cells which are indistinguishable, and (b) occupies a fixed position in space. As there is one factor $N_{0} n ! Q_{n}$ for each rouleau of $n$ cells, one finds for the configuration sum

$$
Q\left\{R_{n}\right\}=\Omega\left\{R_{n}\right\} \prod_{n=1}^{\infty}\left(N_{0} n ! Q_{n}\right)^{R_{n}}
$$

which in combination with (2.3) gives the expression

$$
Q\left\{R_{n}\right\}=N ! \prod_{n=1}^{\infty} \frac{\left(N_{0} Q_{n}\right)^{R_{n}}}{R_{n} !}
$$


The equilibrium distribution $\left\{\boldsymbol{R}_{n}^{*}\right\}$ is found by determining the maximum of $Q\left\{R_{n}\right\}$ subject to the constraint (2.2). As the logarithm is a monotonically increasing function one can as well maximize $\ln Q\left\{R_{n}\right\}$. Using the method of Lagrange multipliers and Stirling's asymptotic formula (cf. Ref. 17) reduces the problem to finding the unconstrained maximum of

$$
L \equiv \sum_{n=1}^{\infty}\left\{R_{n} \ln \left(N_{0} Q_{n}\right)-R_{n} \ln R_{n}+R_{n}-\lambda n R_{n}\right\}
$$

where $\lambda$, the Lagrange multiplier, is a parameter to be determined later. Taking the derivative of $L$ with respect to $R_{n}$ and setting it equal to zero immediately leads to the equilibrium distribution of rouleaux

$$
\frac{R_{n}^{*}}{N_{0}}=Q_{n} \exp (-\lambda n)
$$

The value of the Lagrange multiplier $\lambda$ is determined by substituting the last equation into the constraint equation (2.2), yielding the implicit formula

$$
\frac{N}{N_{0}}=\sum_{n=1}^{\infty} n Q_{n} \exp (-\lambda n)
$$

Note that $N_{0}$ is proportional to the volume $V$. Hence $R_{n}^{*} / N_{0}$, as well as $\lambda$, does not depend on $N$ and $V$ separately, but only on the cell density $N / V$. Finally, one should note that the average number $R_{n, m}^{*}$ of rouleaux with $n$ cells and $m$ branch points follows from (2.8)

$$
\frac{R_{n, m}^{*}}{N_{0}}=Q_{n, m} \exp (-\lambda n)
$$

This is the case because $R_{n, m}^{*} / R_{n}^{*}$ should equal $Q_{n, m} / Q_{n}$, where $Q_{n, m}$ equals the sum of the Boltzmann factors over all those microstates of a single rouleau in which the rouleau (a) consists of $n$ indistinguishable cells, (b) occupies a fixed position in space, and (c) has $m$ branch points.

\section{CALCULATION OF THE CONFIGURATION SUM $Q_{n, m}$}

The considerations in the preceding section led to the formal expressions (2.8)-(2.10) which we shall now make explicit by calculating the configuration sums $Q_{n}$ and $Q_{n, m}$. The basic quantity $Q_{n, m}$ can be calculated in many ways. Here we proceed by making use of the generating function

$$
Q(z, \xi)=\sum_{n=1}^{\infty} \sum_{m=0}^{\infty} Q_{n, m} z^{n} \xi^{m}
$$


where $z$ and $\xi$ are dummy complex variables chosen with $|z|$ and $|\xi|$ small enough so that the sum converges. To determine this generating function we shall follow the method of $G \bar{o}^{(22)}$, which has also been used recently by Wiegel ${ }^{(23)}$. This method is much simpler than the competing methods of Hijmans $^{(24)}$ and de Gennes ${ }^{(25)}$.

In order to count rouleaux which are fixed in space, we shall start the enumeration with one specific cell, fixed in space. The free face of this specifically chosen cell shall be called the root of the rouleau. The rouleau can then be thought of as growing by a cell attaching to the face opposite the root. This choice of notation is motivated by that of graph theory. To represent a rouleau as a graph, each cell is drawn as a branch or edge of the graph. The two nodes which terminate each branch can be thought of as the faces of the cell. Since we only consider rouleaux without loops, the graph will be a tree. A rooted tree has one node, its root, distinguished from the others ${ }^{(26)}$. In our considerations the root will always be an end point of the graph. We thus are dealing with a subclass of rooted trees called planted trees. (A planted tree is a tree rooted at an end point ${ }^{(26)}$.) The relationship between the number of "rooted rouleaux" and the number of rouleaux without a root shall be discussed later in this section. In what follows we shall begin our counting with a single cell and not specify which face is chosen as the root. Since there are two choices we shall count only one half the number of rooted rouleaux and then correct for this at the end of the calculation. Once a root is chosen the use of the Boltzmann factors $\alpha$ and $\beta$ rather than $\alpha^{\prime}$ and $\beta^{\prime}$ will automatically take care of the other degeneracies caused by cells having two faces.

The basic observation which we use to count rooted rouleaux is that every rouleau either contains no branch points at all, or it consists of a straight segment (a cylindrical stack of red cells) followed by a branch point to which are attached two rouleaux with or without branch points. We shall call a rouleau without any branch points a rouleau of order 0 . Let $Q_{0}(z)$ denote the generating function for rouleaux of order 0 , i.e.,

$$
Q_{0}(z)=\sum_{n=1}^{\infty} Q_{n, 0} z^{n}
$$

Since a stack of $n$ cells has $n-1$ adhesive interfaces each with energy $E_{A}$, and each cell can adhere to the next with either of two faces

$$
Q_{n, 0}=\alpha^{n-1}
$$

where $\alpha$ is the weighted Boltzmann factor defined in (1.2). Therefore

$$
Q_{0}(z)=\sum_{n=1}^{\infty} \alpha^{n-1} z^{n}=z(1-\alpha z)^{-1}
$$


Next we consider a larger class of rouleaux, those of order 1. A rouleaux of order 1 starts with a straight segment, which may, but need not, be followed by a branch point, with weighted Boltzmann factor $\beta$, to which are attached two rouleaux of order 0 . Denoting by $Q_{1}(z, \xi)$ the generating function for rouleaux of order 1 , one finds

$$
Q_{1}(z, \xi)=Q_{0}(z)\left\{1+\beta \xi Q_{0}^{2}(z)\right\}
$$

The term 1 between the brackets in this equation reflects the fact that rouleaux of order 0 are included in the class of rouleaux of order 1 . The second term within the brackets is contributed by those rouleaux with a single branch point having weighted Boltzmann factor $\beta$. The variable $\xi$ counts branch points and the factor $Q_{0}^{2}(z)$ arises from the fact that two rouleaux of order zero emanate from the branch point (see Fig. 3).

Generally, a rouleau of order $k$ starts with a straight segment which can bifurcate into two rouleaux of orders at most equal to $k-1$. The generating function $Q_{k}(z, \xi)$ for rouleaux of order $k$ is therefore given by

$$
Q_{k}(z, \xi)=Q_{0}(z)\left\{1+\beta \xi Q_{k-1}^{2}(z, \xi)\right\}
$$

To see that this formula is correct, note from Fig. 3c that there are five possible structures for a rouleau of order 2 . Thus the generating function for rouleaux of order 2 derived either from the figure or from (3.6) is

$$
Q_{2}(z, \xi)=Q_{0}(z)+\beta \xi Q_{0}^{3}(z)+2 \beta^{2} \xi^{2} Q_{0}^{5}(z)+\beta^{3} \xi^{3} Q_{0}^{7}(z)
$$

where in each term, representing a possible structure, there is one factor $\beta \xi$ for each branch point and a factor $Q_{0}(z)$ for each straight segment (branch) in the treelike diagrams representing the rouleaux of order 2 .

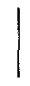

(a)

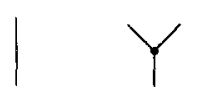

(b)
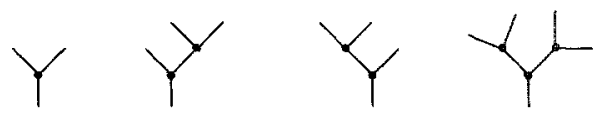

(c)

Fig. 3. Graphical representation of rouleaux of different order. (a) Rouleau of order 0. (b) Rouleaux of order 1. (c) Rouleaux of order 2. 
In the limit $k \rightarrow \infty$ all rouleaux are included automatically in this enumeration process, i.e.,

$$
\lim _{k \rightarrow \infty} Q_{k}(z, \xi)=Q(z, \xi)
$$

Taking this limit (3.6) becomes

$$
Q(z, \xi)=Q_{0}(z)\left\{1+\beta \xi Q^{2}(z, \xi)\right\}
$$

As this is a quadratic equation for the unknown function $Q$ an explicit solution is possible and leads to

$$
Q(z, \xi)=\left(2 \beta \xi Q_{0}\right)^{-1}\left[1-\left(1-4 \beta \xi Q_{0}^{2}\right)^{1 / 2}\right]
$$

Note that the second solution of (3.8), which would have the form of (3.9) but with a + sign in front of the square root, can be disregarded because this solution does not have the property $\lim _{z \rightarrow 0} Q(z, \xi)=0$, which follows from the definition (3.1) of $Q(z, \xi)$ as a power series.

The transformation (3.1) which maps the real numbers $Q_{n, m}$ into the function $Q(z, \xi)$ can be inverted by performing a contour integral around the origin of the complex $\xi$ plane, followed by a contour integral around the origin of the complex $z$ plane. ${ }^{(27)}$ In both cases the contours must have radii small enough so that the sum in (3.1) converges. With this restriction

$$
Q_{n, m}=(2 \pi i)^{-2} \oint \frac{d z}{z^{n+1}} \oint \frac{d \xi}{\xi^{m+1}} Q(z, \xi)
$$

The $\xi$ integral can be performed by noting that the quantity

$$
(2 \pi i)^{-1} \oint Q(z, \xi) \xi^{-m-1} d \xi
$$

equals the coefficient of $\xi^{m}$ in $Q(z, \xi)$. Using (3.9) and the binomial expansion

$$
\begin{aligned}
\left(1-4 \beta \xi Q_{0}^{2}\right)^{1 / 2}= & 1-\frac{1}{2}\left(4 \beta \xi Q_{0}^{2}\right)-\frac{1 \cdot 1}{2 \cdot 4}\left(4 \beta \xi Q_{0}^{2}\right)^{2} \\
& -\frac{1 \cdot 1 \cdot 3}{2 \cdot 4 \cdot 6}\left(4 \beta \xi Q_{0}^{2}\right)^{3}-\cdots
\end{aligned}
$$

one finds

$$
(2 \pi i)^{-1} \oint Q(z, \xi) \xi^{-m-1} d \xi=2 Q_{0} \frac{1 \cdot 1 \cdot 3 \cdot 5 \cdots(2 m-1)}{2 \cdot 4 \cdot 6 \cdot 8 \cdots(2 m+2)}\left(4 \beta Q_{0}^{2}\right)^{m}
$$

Substituting this into (3.10) yields

$$
Q_{n, m}=2(4 \beta)^{m} \frac{1 \cdot 1 \cdot 3 \cdot 5 \cdots(2 m-1)}{2 \cdot 4 \cdot 6 \cdot 8 \cdots(2 m+2)}(2 \pi i)^{-1} \oint z^{-n-1} Q_{0}^{2 m+1} d z
$$


In the same way, the $z$ integral can be calculated using (3.4) and the binomial expansion for $(1-\alpha z)^{-2 m-1}$. This gives the final result

$$
\begin{aligned}
Q_{n, m}= & 2(4 \beta)^{m} \alpha^{n-2 m-1} \frac{1 \cdot 1 \cdot 3 \cdot 5 \cdots(2 m-1)}{2 \cdot 4 \cdot 6 \cdot 8 \cdots(2 m+2)} \\
& \times \frac{(2 m+1)(2 m+2) \cdots(n-1)}{(n-2 m-1) !}
\end{aligned}
$$

which can also be written as

$$
Q_{n, m}=\frac{\beta^{m} \alpha^{n-2 m-1}\left(\begin{array}{c}
n-1 \\
2 m
\end{array}\right)\left(\begin{array}{c}
2 m \\
m
\end{array}\right)}{m+1}
$$

We end this section with three comments. First, we verify that (3.14) has the right qualitative properties. Because there are $m$ branch points in all the rouleaux which are included in $Q_{n, m}$ there should be a Boltzmann factor $\beta^{m}$. This is in agreement with (3.14). Next note that $n$ cells have $2 n$ cell "faces," of which $3 m$ are involved in forming the $m$ branch points and $m+2$ form free ends. Hence $2 n-3 m-m-2=2 n-4 m-2$ cell "faces" form $n-2 m-1$ cell-cell contacts, which should lead to a Boltzmann factor $\alpha^{n-2 m-1}$, also in agreement with (3.14). Lastly, note that when $m=0$ (3.14) reduces to (3.3).

Second, recall that the configuration sum (3.14) must be multiplied by a factor of 2 which counts the number of ways the first red blood cell can be oriented, i.e., the root can be chosen in two ways. Thus the configuration sum of the rooted rouleaux, $Q_{n, m}^{r}$, is given by

$$
Q_{n, m}^{r}=2 Q_{n, m}
$$

Third, observe that when one counts rooted rouleaux one overcounts the number of distinct structures that would be generated by red cell aggregation since each choice of a root gives rise to a different rooted rouleau. For example, if one examines the rouleau represented as a tree in Fig. 4, in which the root is fixed in the location labeled with an $A$, notice that choosing $B, C$, or $D$ as the root gives rise to the same tree, only rotated. Since we "grow" the tree from the root, only ends can be chosen as roots. A tree with $m$ branch points has $m+2$ ends; thus we should divide $Q_{n, m}^{r}$ by $m+2$. To correctly account for rigid rotations of the rouleau about its center of mass we need to introduce a factor of $4 \pi$. Hence it is more accurate to replace (3.15) by

$$
\tilde{Q}_{n, m}=4 \pi(m+2)^{-1} Q_{n, m}^{r}
$$

In this approximation we still have neglected kinetic degrees of freedom of 

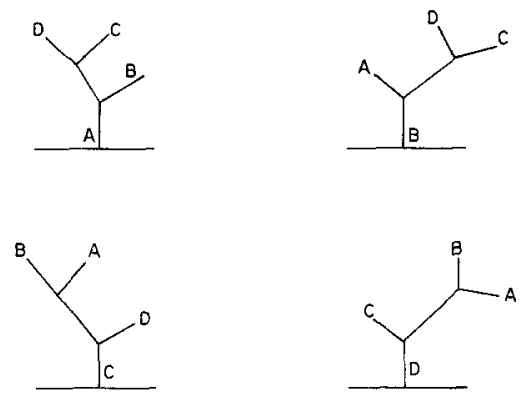

Fig. 4. A graphical representation of rooted rouleaux. Choosing the root as any of the four end points gives rise to the same rouleau only rotated.

the rouleau, for example, elastic vibrations of the rouleau structure. In the rest of this paper we shall continue to use expression (3.14) but occasionally also refer to the consequences of using (3.16).

Fourth, (3.15) and (3.16) together with (2.9) and (2.10) leads to explicit results for $R_{n, m}^{*}$ and $\tilde{R}_{n, m}$ the number of rooted and unrooted rouleaux with $n$ cells and $m$ branch points, respectively. As this is the most "fine grained" type of information about the distribution, all the other quantities of interest, which were enumerated at the end of Section 1, can be derived from it in a fairly straightforward way.

\section{THE DISTRIBUTION OF ROOTED ROULEAUX}

The configuration sum $Q_{n}$, determines through (2.8), (2.9), $R_{n}^{*}$, the distribution of rouleaux with $n$ cells. In principle, $Q_{n}$ can be found from the result of the last section by summation

$$
Q_{n}^{r}=\sum_{m=0}^{\infty} Q_{n, m}^{r}
$$

where we have included the superscript $r$ to denote we have restricted our attention to rooted rouleaux. Similarly, $Q^{r}(z, \xi)$ will denote the generating function with coefficients $Q_{n, m}^{r}$. In Section 6 we numerically calculate $Q_{n}^{r}$ in this way for some specific values of the parameters $n, \alpha$, and $\beta$. However, we have been unable to perform the summation analytically and have therefore turned to alternative methods of determining $Q_{n}^{r}$.

The generating function for $Q_{n}^{r}$,

$$
q^{r}(z) \equiv \sum_{n=1}^{\infty} Q_{n}^{r} z^{n}
$$


for rooted rouleaux can be found by substituting $\xi=1$ into (3.9) and multiplying by two to convert $Q_{n, m}$ to $Q_{n, m}^{r}$. This leads to

$$
q^{r}(z)=\left(\beta Q_{0}\right)^{-1}\left[1-\left(1-4 \beta Q_{0}^{2}\right)^{1 / 2}\right]
$$

which with the substitution of (3.4) for $Q_{0}(z)$ becomes the explicit function of $z$

$$
q^{r}(z)=(\beta z)^{-1}(1-\alpha z)\left\{1-\left[1-\frac{4 \beta z^{2}}{(1-\alpha z)^{2}}\right]^{1 / 2}\right\}
$$

The coefficients $Q_{n}^{r}$ in (4.2) can be determined by taking the Taylor series expansion of $q^{r}(z)$ around $z=0$. The values of $Q_{n}^{r}$ found in this way are listed in Table I. For large values of $n$ this explicit approach becomes prohibitively difficult and the following approximate method can be used.

As $q$ is a power series with positive coefficients it is a monotonically increasing function of $z$ along the positive real $z$ axis. In the complex $z$ plane $q(z)$ has branch points at the solutions of the equation $Q_{0}^{2}(z)$ $=(4 \beta)^{-1}$; these are

$$
z_{0}=(\alpha+2 \sqrt{ } \beta)^{-1} \ll 1
$$

and

$$
z_{\mathrm{I}}=(\alpha-2 \sqrt{ } \beta)^{-1}
$$

Both branch points are situated on the real $z$ axis; $z_{0}$ is closer to the origin than the pole $\alpha^{-1}$ of $Q_{0}(z) ; z_{1}$ is always farther from the origin than $z_{0}$.

In order to calculate $Q_{n}^{r}$ one can integrate $q^{r}(z) z^{-n-1}$ along a contour surrounding the origin in the complex plane. Using the saddle point

Table I. The Boltzmann Weighted Configuration Sum of Rooted Trees, $Q_{n}^{r}$, for Small Values of $n$

\begin{tabular}{rl}
\hline$n$ & \multicolumn{1}{c}{$Q_{n}^{r} / 2$} \\
\hline 1 & 1 \\
2 & $\alpha$ \\
3 & $\alpha^{2}+\beta$ \\
4 & $\alpha^{3}+3 \alpha \beta$ \\
5 & $\alpha^{4}+6 \alpha^{2} \beta+2 \beta^{2}$ \\
6 & $\alpha^{5}+10 \alpha^{3} \beta+10 \alpha \beta^{2}$ \\
7 & $\alpha^{6}+15 \alpha^{4} \beta+30 \alpha^{2} \beta^{2}+5 \beta^{3}$ \\
8 & $\alpha^{7}+21 \alpha^{5} \beta+70 \alpha^{3} \beta^{2}+35 \alpha \beta^{3}$ \\
9 & $\alpha^{8}+28 \alpha^{6} \beta+140 \alpha^{4} \beta^{2}+140 \alpha^{2} \beta^{3}+14 \beta^{4}$ \\
10 & $\alpha^{9}+36 \alpha^{7} \beta+252 \alpha^{5} \beta^{2}+420 \alpha^{3} \beta^{3}+126 \alpha \beta^{4}$ \\
\hline
\end{tabular}


method $^{(18,28)}$ to approximate this integral, one finds that for large $n$ the major contribution to the integral occurs in the neighborhood of $z_{0}$, the singularity nearest the origin. Knowing this it suffices to expand the function under the square root in (4.3) in the vicinity of $z_{0}$

$$
1-4 \beta Q_{0}^{2}(z)=a^{2}\left(z_{0}-z\right)+O\left(z_{0}-z\right)^{2}
$$

where

$$
a^{2} \equiv 8 \beta Q_{0}\left(z_{0}\right) Q_{0}^{\prime}\left(z_{0}\right)=4 \sqrt{\beta}\left(1+\frac{\alpha}{2 \sqrt{ } \beta}\right)^{2}
$$

Close to $z=z_{0}$ one has, therefore, the power series expansion

$$
\begin{aligned}
q^{r}(z) \cong\left[\beta Q_{0}\left(z_{0}\right)\right]^{-1} a z_{0}^{1 / 2}\{ & \frac{1}{2}\left(\frac{z}{z_{0}}\right)+\frac{1 \cdot 1}{2 \cdot 4}\left(\frac{z}{z_{0}}\right)^{2} \\
& \left.+\frac{1 \cdot 1 \cdot 3}{2 \cdot 4 \cdot 6}\left(\frac{z}{z_{0}}\right)^{3}+\cdots\right\}
\end{aligned}
$$

The configuration sum $Q_{n}^{r}$, which is given by the coefficient of $z^{n}$ in this power series, is now found to equal

$$
Q_{n}^{r} \cong 2 a \beta^{-1 / 2} z_{0}^{1 / 2-n} \frac{1 \cdot 1 \cdot 3 \cdot 5 \cdots(2 n-3)}{2 \cdot 4 \cdot 6 \cdot 8 \cdots 2 n} \quad(n \gg 1)
$$

where we have used the fact that $Q_{0}\left(z_{0}\right)=(4 \beta)^{-1 / 2}$. The product on the right-hand side can be calculated with the formula of Wallis icf. equation (6.1.49) of Ref. 29\}. In this way one finds

$$
Q_{n}^{r} \cong c n^{-3 / 2} z_{0}^{-n} \quad(n \gg 1)
$$

where

$$
c \equiv a(\pi \beta)^{-1 / 2} z_{0}^{1 / 2}=\left[\left(\frac{2}{\pi \beta}\right)\left(1+\frac{\alpha}{2 \sqrt{ } \beta}\right)\right]^{1 / 2} \ll 1
$$

The same result (4.10) can also be derived by evaluating a contour integral around the origin of the complex $z$ plane with the saddlepoint method in the case $n \gg 1$.

The equilibrium distribution of the rouleaux now follows from (2.8), (2.9), and (4.11) by eliminating $\mu$ between

$$
\frac{R_{n}^{*}}{N_{0}} \cong c n^{-3 / 2} \mu^{n}
$$

and

$$
\frac{N}{N_{0}} \cong c \sum_{n=1}^{\infty} n^{-1 / 2} \mu^{n}
$$


where

$$
\mu \equiv z_{0}^{-1} \exp (-\lambda)=(\alpha+2 \sqrt{\beta}) \exp (-\lambda)
$$

Note that the two energies $E_{A}$ and $E_{B}$ only enter the distribution in the combination (4.12) which defines the constant $c$. Of special interest is the regime where $N$ and $N_{0}$ are of the same order of magnitude, $c \ll 1$ and $n \gg 1$. In this regime the value of $\mu$ must be close to (but slightly smaller than) unity in order to give the series $\sum n^{-1 / 2} \mu^{n}$ the large value $N / c N_{0}$. When $\mu=1$ the series diverges, but it converges when $\mu<1$. Hence the rouleau distribution can be approximated by

$$
\frac{R_{n}^{*}}{N_{0}} \cong c n^{-3 / 2}
$$

This is the main result of the present theory. It is remarkably simple and subject to experimental verification. It shows that $R_{n}^{*}$ is always a decreasing function of $n$, which means that a larger rouleau is always less probable than a smaller one. The distribution nevertheless has a very long "tail" due to the slow decay of the factor $n^{-3 / 2}$.

A more precise estimate of the rouleau distribution can be obtained by solving (4.14) for $\mu$. This equation involves a summation of the form

$$
\phi(\mu, s)=\sum_{n=1}^{\infty} n^{-s} \mu^{n}
$$

studied by Truesdel1 ${ }^{(30)}$ for $s=1 / 2,3 / 2$, and $-1 / 2$. For $\mu$ near, but smaller than one, the asymptotic formula ${ }^{(30)}$

$$
\phi(\mu, s)=\Gamma(1-s)(-\ln \mu)^{s-1}+\sum_{n=0}^{\infty} \zeta(s-n) \frac{(\ln \mu)^{n}}{n !}
$$

can be used. Here $\Gamma(\cdot)$ denotes the gamma function and $\zeta(\cdot)$ is Riemann's zeta function. The values of both functions are tabulated in Dwight. ${ }^{(31)}$ With $s=1 / 2,(4.18)$ becomes

$$
\phi\left(\mu, \frac{1}{2}\right) \cong \sqrt{\pi}(-\ln \mu)^{-1 / 2}+\zeta\left(\frac{1}{2}\right)+\zeta\left(-\frac{1}{2}\right) \ln \mu
$$

where we have omitted terms of order $(\ln \mu)^{2}$ and higher; $\zeta(1 / 2) \cong-1.46$ compared to which the omitted terms are negligible when $\mu$ is near 1 .

The substitution of (4.19) into (4.14) yields an equation, which when squared and terms of order $(\ln \mu)^{2}$ neglected can be solved to yield

$$
\mu=\exp \left\{\frac{-\pi}{b^{2}}\left[1-\frac{2 \pi \zeta(-1 / 2)}{b^{3}}\right]\right\}
$$


where

$$
b \equiv \frac{N}{c N_{0}}-\zeta\left(\frac{1}{2}\right)
$$

If $N / c N_{0} \gg 1$, then the simple expression

$$
\mu=\exp \left[-\pi\left(\frac{c N_{0}}{N}\right)^{2}\right] \cong 1-\pi\left(\frac{c N_{0}}{N}\right)^{2}
$$

suffices.

The Lagrange multiplier $\lambda$ now follows directly from (4.15) and (4.20),

$$
\lambda=\ln (\alpha+2 \sqrt{\beta})+\frac{\pi}{b^{2}}\left[1-\frac{2 \pi \zeta(-1 / 2)}{b^{3}}\right]
$$

which in the case $N / C N_{0} \gg 1$ simplifies to

$$
\lambda=\ln (\alpha+2 \sqrt{\beta})+\pi\left(\frac{c N_{0}}{N}\right)^{2}
$$

Equations (4.20) and (4.21) are only approximate because they depend upon the asymptotic result (4.11) and the asymptotic formula (4.19). The Lagrange multiplier $\lambda$ may be determined exactly using the generating function $q^{r}(z)$. From the definition of $q^{r}(z)$ as a power series, (4.2), one sees that (2.9), which determines $\lambda$, is equivalent to the following equation:

$$
\frac{N}{N_{0}}=\left(z \frac{\partial q^{r}}{\partial z}\right)_{z=\exp (-\lambda)}
$$

where the expression on the right is evaluated at $z=\exp (-\lambda)$ after the differentiation is performed. Evaluating the derivative in (4.22) through the use of (4.4), results in the following implicit equation for $\lambda$ :

$$
\frac{N}{N_{0}}=\frac{1-\alpha z-\left[(1-\alpha z)^{2}-4 \beta z^{2}\right]^{1 / 2}}{\beta z\left[(1-\alpha z)^{2}-4 \beta z^{2}\right]^{1 / 2}}
$$

where $z=\exp (-\lambda)$. As we show in Section 6, this equation can easily be solved by numerical methods. Because $N / N_{0}$ is real, $(1-\alpha z)^{2}>4 \beta z^{2}$ or $z<z_{0}=(\alpha+2 \sqrt{\beta})^{-1}$. Therefore $\lambda<-\ln z_{0}$. Given a value of $\lambda$ that solves (4.23), $\mu$ can be determined exactly from (4.15).

It is easy to calculate other quantities of interest. For example, using (2.13) and (3.1) the average number of cells per rouleau can be expressed in terms of the generating function $Q(z, \xi)$

$$
\langle n\rangle \equiv \frac{\sum_{n, m} n R_{n, m}^{*}}{\sum_{n, m} R_{n, m}^{*}}=\left(\frac{z}{Q} \frac{\partial Q}{\partial z}\right)_{z=\exp (-\lambda), \xi=1}
$$


Since we are not differentiating with respect to $\xi$, we can set $\xi=1$ before differentiating and hence replace $Q(z, 1)$ by $q^{r}(z)$. If we now substitute (4.22) into (4.24) we obtain $\langle n\rangle_{r}$, the average number of cells per rooted rouleau:

$$
\langle n\rangle_{r}=\frac{N}{N_{0} q^{r}[\exp (-\lambda)]}
$$

which shows that the average number of cells per rouleau grows in proportion to the total number of cells, $N$. Alternatively, the substitution of (4.4), (1.1)-(1.3), and (4.23) into (4.25) yields a form which illustrates the dependence of $\langle n\rangle_{r}$ on the energies $E_{A}$ and $E_{B}$ :

$$
\langle n\rangle_{r}=\left\{\left[1-2 \exp \left(E_{A} / k_{B} T-\lambda\right)\right]^{2}-16 \exp \left(E_{B} / k_{B} T-2 \lambda\right)\right\}^{-1 / 2}
$$

For the calculation of other statistical properties of rouleaux one can make use of the following relations which, for any rouleau, connect the number $(S)$ of straight segments, the number $(I)$ of internal straight segments, and the number $(E)$ of external straight segments with the number $(m)$ of branch points

$$
\begin{aligned}
& S=2 m+1 \\
& I=m-1 \\
& E=m+2
\end{aligned}
$$

where $m=0,1,2,3, \ldots$ It is, therefore, only necessary to calculate the average number $\langle m\rangle$ of branch points per rouleau. Rather than giving full details we sketch the outline of the calculation of this average. Using (2.10) and (3.1) one finds

$$
\langle m\rangle \equiv \frac{\sum_{n, m} m R_{n, m}^{*}}{\sum_{n, m} R_{n, m}^{*}}=\left(\frac{\xi}{Q} \frac{\partial Q}{\partial \xi}\right)_{z=\exp (-\lambda), \xi=1}
$$

\section{THE DISTRIBUTION OF UNROOTED ROULEAUX}

It was already noticed at the end of Section 3 that $Q_{n, m}^{r}$ as given by (3.15) represents the configuration sum over all rooted rouleaux of $n$ cells with $m$ branch points. It is probably somewhat more realistic to represent rouleaux by unrooted trees than by rooted trees. Rather than mimic the analysis in Section 4, we shall use a different method to find the asymptotic distribution of unrooted rouleaux when $n \gg 1$. Let $\tilde{R}_{n}^{*}$ and $\tilde{R}_{n, m}^{*}$ be the numbers of unrooted rouleaux with $n$ cells, and with $n$ cells and $m$ branch points, respectively, in a state of thermal equilibrium. Using (2.10) and 
(3.16) one finds

$$
\tilde{R}_{n}^{*}=\sum_{m} \tilde{R}_{n, m}^{*}=d_{n} \sum_{m}(m+2)^{-1} Q_{n, m}^{r}
$$

where

$$
d_{n}=4 \pi N_{0} \exp (-\lambda n)
$$

Having calculated in the last section the number of rooted rouleaux, $R_{n}^{*}$, it is expedient to express $\tilde{R}_{n}^{*}$ in terms of $R_{n}^{*}$. To do so we write (5.1) as

$$
\tilde{R}_{n}^{*}=d_{n} \sum_{m}\left(\langle m\rangle_{n}+2\right)^{-1}\left(1+\frac{m-\langle m\rangle_{n}}{\langle m\rangle_{n}+2}\right)^{-1} Q_{n, m}^{r}
$$

where $\langle m\rangle_{n}$ is the average number of branch points of all rooted rouleaux which consist of $n$ cells. Equation (5.3) can be expressed in terms of the series

$$
\begin{aligned}
\tilde{R}_{n}^{*} & =d_{n} \sum_{m}\left(\langle m\rangle_{n}+2\right)^{-1} Q_{n, m}^{r}\left[1-\left(\frac{m-\langle m\rangle_{n}}{\langle m\rangle_{n}+2}\right)+\left(\frac{m-\langle m\rangle}{\langle m\rangle_{n}+2}\right)^{2}-\cdots\right] \\
& =S_{0}-S_{1}+S_{2}-\cdots
\end{aligned}
$$

where the sum

$$
S_{i}=d_{n} \sum_{m}\left(\langle m\rangle_{n}+2\right)^{-1} Q_{n, m}^{r}\left(\frac{m-\langle m\rangle_{n}}{\langle m\rangle_{n}+2}\right)^{i}
$$

Observe $S_{1}=0$ since

$$
\langle m\rangle_{n}=\frac{\sum_{m} m \tilde{R}_{n, m}^{*}}{\sum_{m} \tilde{R}_{n, m}^{*}}=\frac{\sum_{m} m Q_{n, m}^{r}}{\sum_{m} Q_{n, m}^{r}}
$$

Also

$$
\frac{S_{2}}{S_{0}}=\frac{1}{\left(\langle m\rangle_{n}+2\right)^{2}} \frac{\sum_{m}\left(m-\langle m\rangle_{n}\right)^{2} Q_{n, m}^{r}}{\sum_{m} Q_{n, m}^{r}}=\frac{\left\langle m^{2}\right\rangle_{n}-\langle m\rangle_{n}^{2}}{\left(\langle m\rangle_{n}+2\right)^{2}}
$$

where $\left\langle m^{2}\right\rangle_{n}-\langle m\rangle_{n}^{2}$ is the variance in the number of branch points of rooted rouleaux with $n$ cells. If this variance is small, or if $\langle m\rangle_{n}$ is large as would be the case when $n, m \gg 1$, then $S_{2} / S_{0} \ll 1$ and

$$
\tilde{R}_{n}^{*}=4 \pi\left(\langle m\rangle_{n}+2\right)^{-1} R_{n}^{*}
$$

In order to find the distribution of unrooted rouleaux in terms of the distribution of rooted rouleaux it is now necessary to calculate the average number $\langle m\rangle_{n}$ of branch points of all rooted rouleaux which consist of $n$ 
cells. This can most easily be done by noticing that for large rouleaux $\langle m\rangle_{n}$ will be very close to that value of $m$ for which $Q_{n, m}^{r}$ attains its maximum. At the maximum

$$
\frac{Q_{n, m}}{Q_{n, m+1}}=1
$$

Substituting (3.14) this condition becomes

$$
(n-2 m-1)(n-2 m-2)=\beta^{-1} \alpha^{2}(m+2)(m+1)
$$

hence

$$
\langle m\rangle_{n} \cong \frac{n}{2+(\alpha / \sqrt{ } \beta)} \quad(n, m \gg 1)
$$

The distribution of unrooted rouleaux for $n \gg 1$ is now found by substitution of the last equation into (5.9), noticing that $\langle m\rangle_{n} \gg 2$, and using (4.13). This procedure gives

$$
\frac{\tilde{R}_{n}^{*}}{N_{0}} \cong 4 \pi c\left(2+\frac{\alpha}{\sqrt{ } \beta}\right) n^{-5 / 2} \mu^{n} \quad(n \gg 1)
$$

We close this section with some general comments on this remarkably simple result. First, it is seen from (5.12) that the average number of cells in a straight segment is given by

$$
\frac{n}{S}=\frac{n}{2\langle m\rangle_{n}+1} \cong 1+\frac{\alpha}{2 \sqrt{ } \beta} \quad(n \gg 1)
$$

where (4.27a) was used. When rouleaux are broken up into straight segments by hydrodynamic processes such as shearing, the individual segments should on the average consist of $1+(\alpha / 2 \sqrt{ } \beta)$ cells.

Second, it is seen that the distributions (4.16) and (5.13) of rooted and unrooted rouleaux differ in one important qualitative respect. The series

$$
N_{u} \equiv \sum_{n=1}^{\infty} n \tilde{R}_{n}^{*} \cong 4 \pi N_{0} c\left(2+\frac{\alpha}{\sqrt{ } \beta}\right) \sum_{n=1}^{\infty} n^{-3 / 2} \mu^{n}
$$

converges for all values of $\mu \leqslant 1$, but diverges for $\mu>1$. Therefore, for unrooted rouleaux $\mu \leqslant 1$. As $\mu$ increases from zero, $N_{u}$ increases and obtains a maximum value when $\mu=1$. Consequently, for some values of the parameters $\alpha$ and $\beta$ only a finite fraction of the total number of red cells can be accommodated in unrooted rouleaux of finite size. This number $N_{u, \max }$ is given by the right-hand side of (5.15) with $\mu=1$ and the sum replaced by its value, $\phi(1,3 / 2) \cong 2.612$,

$$
N_{u, \max } \cong 32.8 N_{0} c[2+(\alpha / \sqrt{ } \beta)]
$$


The remaining number of red cells,

$$
N_{c} \equiv N-N_{u, \max }
$$

will presumably condense into a single macroscopic clot which does not have a treelike structure and which might flocculate out of the solution. This situation, which is reminiscent of the phenomenon of Bose condensation in an ideal gas of Bose particles, ${ }^{(17,18)}$ and of the phenomenon of gelation in a system of self-aggregating multivalent particles ${ }^{(32)}$ indicates the breakdown of our assumption that the rouleaux are treelike structures at large hematocrits, and deserves further research.

When the system parameters lie outside the region that corresponds to condensation, $\mu<1$. As in Section 4, one can estimate $\mu$ using the Truesdell function $\phi(\mu, 3 / 2)$, or compute $\lambda$ and then $\mu$ from the red cell conservation law which can be written as a modified form of (4.22), i.e.,

$$
\frac{N}{N_{0}}=\left(z \frac{\partial Q^{u}}{\partial z}\right)_{z=\exp (-\lambda), \xi=1}
$$

where the generating function

$$
Q^{u}(z, \xi) \equiv \sum_{n} \sum_{m} \tilde{Q}_{n, m} z^{n} \xi^{m}
$$

Using (3.16), (4.1), and (4.2) it is easy to see that

$$
\frac{1}{4 \pi \xi} \frac{d}{d \xi}\left[\xi^{2} Q^{u}(z, \xi)\right]=Q^{r}(z, \xi)
$$

from which one can conclude

$$
Q^{u}(z, \xi)=4 \pi\left[6 \beta^{2} \xi^{2} Q_{0}^{3}(z)\right]^{-1}\left\{6 \beta \xi Q_{0}^{2}(z)-1+\left[1-4 \beta \xi Q_{0}^{2}(z)\right]^{3 / 2}\right\}
$$

Substituting this into (5.17) one finds the following implicit equation for $\lambda$ :

$$
\frac{N}{N_{0}}=\left.\frac{4 \pi}{\beta z U(z)}\left\{2-U(z)-2[1-U(z)]^{1 / 2}\right\}\right|_{z=\exp (-\lambda)}
$$

where

$$
U(z) \equiv 4 \beta Q_{0}^{2}(z)
$$

is evaluated at $z=\exp (-\lambda)$. By solving (5.21) for $\lambda$ and substituting into (4.15) one obtains $\mu$. From $Q^{u}(z, \xi)$ one can obtain using (4.24) and (4.28) the average number of cells, $\langle n\rangle_{u}$, and the average number of branch noints. $\langle m\rangle_{u}$, per unrooted rouleaux. These procedures will be used in the numerical calculations discussed in Section 7. 
Equation (5.21) is a modified form of the red cell conservation law. When $U(z)>1$ the right side becomes imaginary and no solution exists. Thus $U(z)=1$ is the condition for condensation. Perelson and Goldstein (manuscript in preparation) show that for condensation polymerization reactions $U(z)=1$ is equivalent to the classical criteria for gelation (cf. Ref. 32). Further at $U(z)=1,\left\langle n^{2}\right\rangle_{u}$ and higher moments all become infinite, as occurs in gelation.

\section{PARAMETER VALUES}

Our theory of equilibrium rouleau sizes depends upon six physically determined parameters: the total number of red cells in the system $N$, the system volume, $V$, the energies of adhesion in a straight segment, $E_{A}$, and at a branch point, $E_{B}$, the absolute temperature $T$, and $N_{0}$, the number of different positions a rouleau can assume within the volume $V$. The temperature and volume will be assumed to be known. If $v$ in (2.4) is chosen as the volume of a red cell then, as we discussed previously, ${ }^{(4)}$

$$
N / N_{0}=N v / V
$$

is the ratio of the volume occupied by red cells to the total system volume, a quantity of physiological significance called the hematocrit. ${ }^{(33)}$ Normal human blood has a hematocrit of approximately $45 \%$ and rouleau experiments are done at this or lower hematocrits. A $1 \%$ hematocrit corresponds to $1.07 \times 10^{5}$ erythrocytes per $\mathrm{mm}^{3(33)}$; thus at this hematocrit $N / V=$ $0.01 N_{0} / V=10^{5} \mathrm{~mm}^{-3}$, or $N_{0} / V \cong 10^{7}$ erythrocytes $\mathrm{mm}^{-3}$. If the hematocrit is $h$, then $N / V=h N_{0} / V$ is determined. In any experiment the hematocrit is known. Consequently, the number of rouleaux at equilibrium, $R_{n}^{*}$ and $R_{n, m}^{*}$, if expressed per unit volume, will depend only upon $E_{A}$ and $E_{B}$, the two basic parameters in our theory.

There have been numerous theoretical and experimental investigations of the energetics of red cell adhesion. Erythrocytes have a negative surface charge. In rouleau formation the electrostatic repulsion between two cells is thought to be overcome by macromolecules which simultaneously adhere to the opposing faces of the adherent cells. ${ }^{(34,35)}$ Thus as reviewed by Chien ${ }^{(34,35)}$ the value of $E_{A}$ will depend upon the concentration of the bridging macromolecules, the number of bonds made with the cell surface per bridging molecule, the interaction energy per bond, the ionic strength of the solution, as well as factors intrinsic to the red cell, including its surface charge, and the elastic properties of the membrane. Consequently, the value of $E_{A}$ may be quite variable depending upon the experimental conditions. For aggregation due to Dextran 70 , with average molecular weight 75,000 daltons, the aggregation energy per unit area has been estimated to be of 
order $10^{-4} \mathrm{ergs} / \mathrm{cm}^{2} .^{(34,35)}$ Since the area of contact between two cells in a rouleau is of order $50 \mu \mathrm{m}^{2}$, at $37^{\circ} \mathrm{C}$ the net adhesion energy $E_{A}$ is of order $10^{3} k_{B} T$. The Boltzmann factor $\alpha$ corresponding to this value of $E_{A}$ is of order $10^{434}$ and thus too large to easily be used in most modern computers. Consequently, in the next section when we numerically evaluate the formulas in our theory we have chosen to use $E_{A}=10 k_{B} T$, a value significantly smaller than estimated by Chien, ${ }^{(34,35)}$ but one that could be attained experimentally at sufficiently low concentrations of the bridging macromolecule. We know of no experimental measurements of $E_{B}$. However, as we argued in Section 1 for a 3-cell branch, $E_{A} \leqslant E_{B} \leqslant 2 E_{A}$. Thus we can estimate a range of $E_{B}$ values from $E_{A}$.

\section{NUMERICAL RESULTS}

In order to gain insight into the predictions of our theory and to test the applicability of our asymptotic results we have numerically evaluated the formulas that determine the rouleau size distribution. We have previously reported ${ }^{(4)}$ some of our numerical results using $Q_{n, m}$ as given by (3.14). Here we will rigorously extend these results to the cases of rooted and unrooted rouleaux.

Our analyses in Sections 3-5 gave rise to explicit expressions for the configuration sums of rooted rouleaux

$$
Q_{n, m}^{r}=\frac{2 \beta^{m} \alpha^{n-2 m-1}\left(\begin{array}{c}
n-1 \\
2 m
\end{array}\right)\left(\begin{array}{c}
2 m \\
m
\end{array}\right)}{m+1}
$$

and unrooted rouleaux (including rigid rotations)

$$
\tilde{Q}_{n, m}=4 \pi(m+2)^{-1} Q_{n, m}^{r}
$$

These quantities determine $R_{n, m}^{*}$ and $\tilde{R}_{n, m}^{*}$, the equilibrium number of rooted and unrooted rouleaux with $n$ cells and $m$ branch points, through the equations

$$
\begin{aligned}
& R_{n, m}^{*}=N_{0} Q_{n, m}^{r} \exp (-\lambda n) \\
& \tilde{R}_{n, m}^{*}=4 \pi N_{0}(m+2)^{-1} N_{0} Q_{n, m}^{r} \exp (-\lambda n)
\end{aligned}
$$

once the Lagrange multiplier $\lambda$ is found. Making use of the generating function $Q^{r}(z, \xi)$ we showed that for rooted rouleaux $\lambda$ obeys the equation

$$
\frac{N}{N_{0}}=\frac{1-\alpha \exp (-\lambda)-\left\{[1-\alpha \exp (-\lambda)]^{2}-4 \beta \exp (-2 \lambda)\right\}^{1 / 2}}{\beta \exp (-\lambda)\left\{[1-\alpha \exp (-\lambda)]^{2}-4 \beta \exp (-2 \lambda)\right\}^{1 / 2}}
$$


The equilibrium number of rouleaux with $n$ cells, $R_{n}^{*}$ or $\tilde{R}_{n}^{*}$, can be determined by summations of the form

$$
R_{n}^{*}=\sum_{m=0}^{[(n-1) / 2]} R_{n, m}^{*}
$$

where $[(n-1) / 2]$ denotes the greatest integer contained in $(n-1) / 2$. The upper limit in this summation is determined by the fact that $Q_{n, m}^{r}=0$ when $2 m>n-1$. To perform the summation analytically one needs to explicitly sum $Q_{n, m}^{r}$ over $m$ to obtain $Q_{n}^{r}$. Being unable to perform this summation, we derived an asymptotic analytical formula for $Q_{n}^{r},(4.11)$, which is valid for $n \gg 1$. Using this approximate form for $Q_{n}^{r}$ we then found

$$
R_{n}^{*} \cong c N_{0} n^{-3 / 2} \mu^{n}, \quad n \gg 1
$$

where

$$
c=\left[\left(\frac{2}{\pi \beta}\right)\left(1+\frac{\alpha}{2 \sqrt{\beta}}\right)\right]^{1 / 2}
$$

and

$$
\mu=(\alpha+2 \sqrt{\beta}) \exp (-\lambda)
$$

Assuming the number of rooted rouleaux with $n$ cells is given by (7.5), we then derived an asymptotic formula for the number of unrooted rouleaux with $n$ cells,

$$
\tilde{R}_{n}^{*} \cong 4 \pi c N_{0}\left(2+\frac{\alpha}{\sqrt{ } \beta}\right) n^{-5 / 2} \mu^{n}, \quad n \gg 1
$$

where $\mu$ is still given by (7.7) but $\lambda$ is now determined by the solution to

$$
\frac{N}{N_{0}}=\frac{4 \pi\left[2-U-2(1-U)^{1 / 2}\right]}{\beta U \exp (-\lambda)}
$$

with

$$
U=4 \beta \exp (-2 \lambda)[1-\alpha \exp (-\lambda)]^{2}
$$

Equations (7.1)-(7.10), which form the basis of our model, were evaluated numerically. In Table II we list values of $Q_{n, m}^{r}$ computed using the recursion relation

$$
Q_{n, m}^{r}=\frac{\beta}{\alpha^{2}} \frac{(n-2 m+1)(n-2 m)}{m(m+1)} Q_{n, m-1}^{r}, \quad m=1,2, \ldots
$$

derived from (7.1a). To start the recursion $Q_{n, 0}^{r}$ is evaluated using (3.3) and (3.15). For the computations shown in the table we chose $E_{A}=10 k_{B} T$ and 


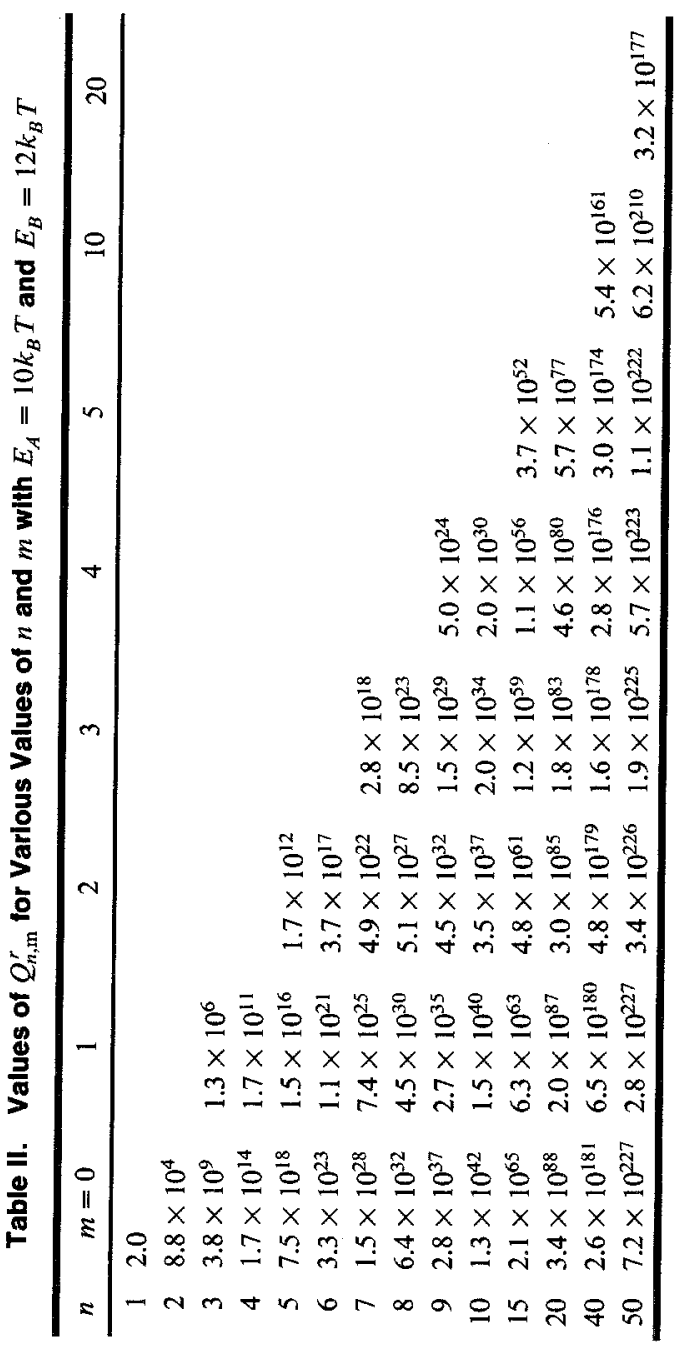


$E_{B}=12 k_{B} T$, i.e., $\alpha=4.4 \times 10^{4}$ and $\beta=6.5 \times 10^{5}$. Even for these small values of $E_{A}$ and $E_{B}$ one sees that $Q_{n, m}^{r}$ grows very rapidly with $n$, as is to be expected because $Q_{n, 0}^{r}=2 \alpha^{n-1}$. For all values of $n$ shown, $Q_{n, 0}^{r}>Q_{n, 1}^{r}$ $>Q_{n, 2}^{r}>\cdots>Q_{n, m}^{r}$ since $\beta / \alpha^{2}=3.4 \times 10^{-4}$ is sufficiently small to counterbalance the increase in configurations that become available as $m$ increases and the trees become more highly branched. However, as $n$ gets large, the configurational term $(n-2 m+1)(n-2 m) / m(m+1)$ will eventually dominate the energetic factor $\beta / \alpha^{2}$ and the configuration sum for some branched trees will be greater than the configuration sum for unbranched structures. For example, when $n \cong\left(2 \alpha^{2} / \beta\right)^{1 / 2}, Q_{n, 1}^{r}>Q_{n, 0}^{r}$. This occurs when $n$ is of order 80 for the parameters used in computing Table II.

Proceeding with the analysis of rooted rouleaux we solved (7.3) for $\lambda$ using an algorithm developed by Dekker ${ }^{(36)}$ to find the zero of a real valued function within a prescribed interval. With $E_{A}$ and $E_{B}$ as given above and the hematocrit $N / N_{0}=0.01, \lambda=10.7673$. When the hematocrit $N / N_{0}=0.05, \lambda=10.7374$ and when $N / N_{0}=0.45, \lambda=10.72945$. Thus there is extremely little variation in the value of $\lambda$ over the hematocrits of experimental interest. This is not surprising for as we argued in Section 4, $\mu \equiv z_{0}^{-1} \exp (-\lambda)$ must be close to, but slightly less than unity. Therefore $\lambda=-\ln \mu z_{0}$ must be slightly larger than $-\ln z_{0}=\ln (\alpha+2 \sqrt{\beta})=10.7291$. For the three values of hematocrit discussed above, $1 \%$, $5 \%$, and $45 \%$, our calculations using (4.23) and (4.15) show $\mu=0.9626,0.9918$, and 0.9997, respectively. Thus the values of $\mu$ are close to, but less than unity, as predicted. Evaluating (4.21a), the approximate formula for $\lambda$, we find $\lambda=11.0167,10.7553$, and 10.72954 , for the $1 \%, 5 \%$, and $45 \%$ hematocrits. Thus this analytic expression yields results accurate to $0.001 \%$ at $45 \%$ hematocrit, but only $2 \%$ accurate at an hematocrit of $1 \%$.

Using a 5\% hematocrit for illustrative purposes, we list in Table III the values of $R_{n, m}^{*}$ for various values of $n$ and $m$, computed using a recursion. scheme based upon (7.2) and (7.10) assuming $E_{A}=10 k_{B} T$ and $E_{B}$ $=12 k_{B} T$. For these values of $E_{A}$ and $E_{B}$ the rouleaux tend not to be highly branched. The average number of branch points, $\langle m\rangle_{r}$, computed over the whole population of rouleaux via (4.28), shows $\langle m\rangle_{r}$ is very small $\left(\langle m\rangle_{r}\right.$ $=0.35$ ). However, as $E_{B}$ becomes larger, branching becomes more probable and $\langle m\rangle_{r}$ increases. In Fig. 5 we show the effect of increasing $E_{B}$, with $E_{A}$ held fixed at $10 k_{B} T$, over the range specified by (1.4) with the hematocrit fixed at $5 \%$.

Computing $\langle n\rangle_{r}$ with (4.26) shows that at $5 \%$ hematocrit with $E_{A}$ $=10 k_{B} T$ and $E_{B}=12 k_{B} T$ the average rouleau size is 39.5 . At $1 \%$ and $45 \%$ hematocrits, the corresponding values are $\langle n\rangle_{r}=15.9$ and 207.9, respectively. Thus the higher the hematocrit the larger the rouleaux. This can be seen more dramatically in Fig. 6, where we plot the concentration of rooted rouleaux of size $n, R_{n}^{*}$, versus $n$ for various hematocrits. 


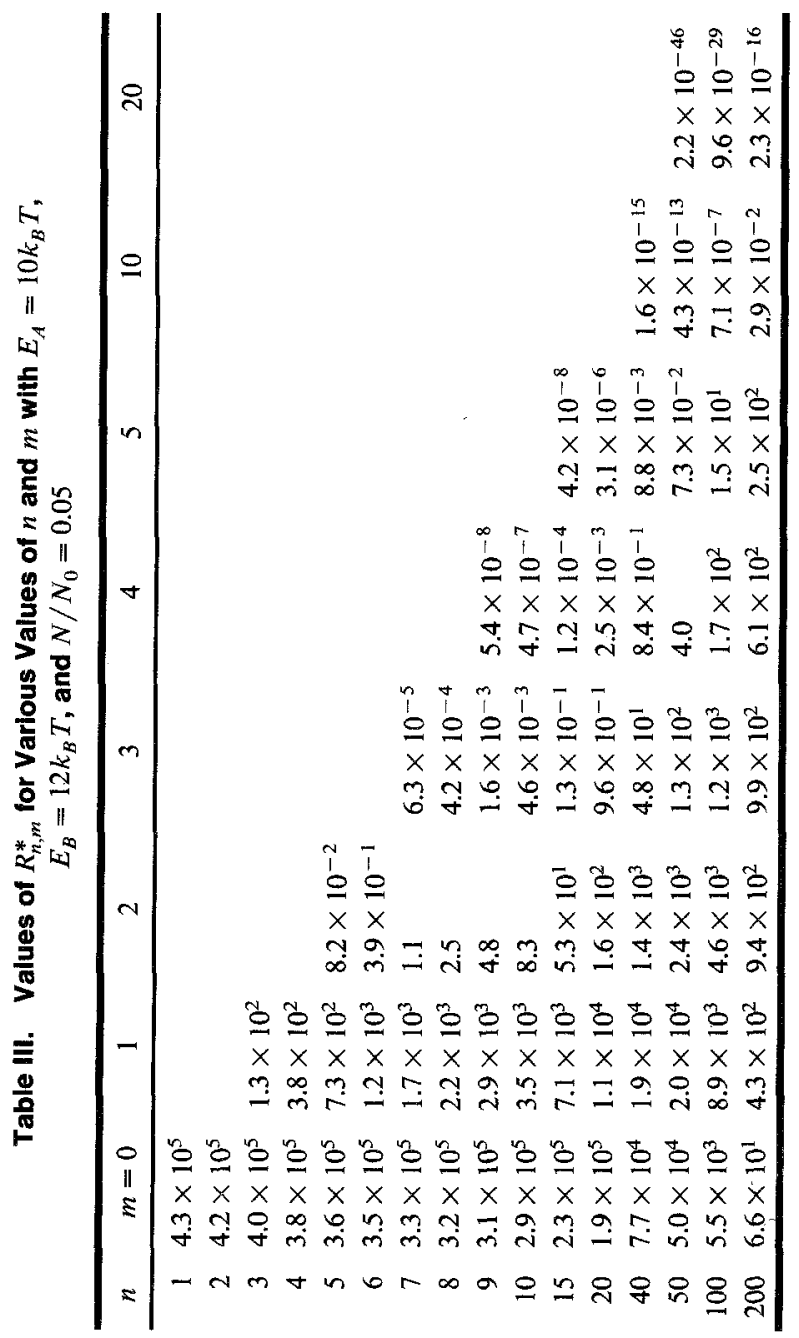




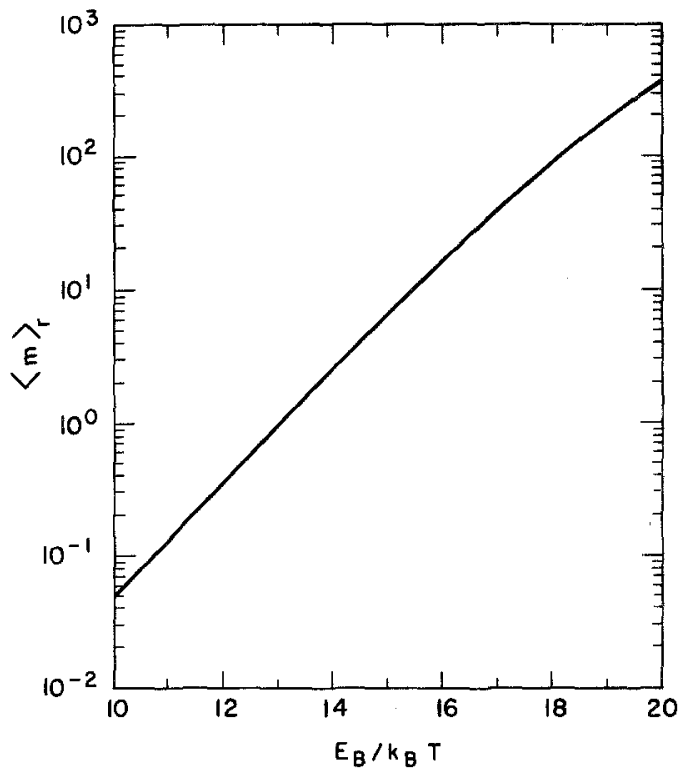

Fig. 5. The mean number of branch points per rooted rouleau, $\langle m\rangle_{r}$, plotted against the branching energy $E_{B}$, with $E_{A}=10 k_{B} T, N_{0}=10^{7}$ cells $/ \mathrm{mm}^{3}$ and $N / N_{0}=5 \%$.

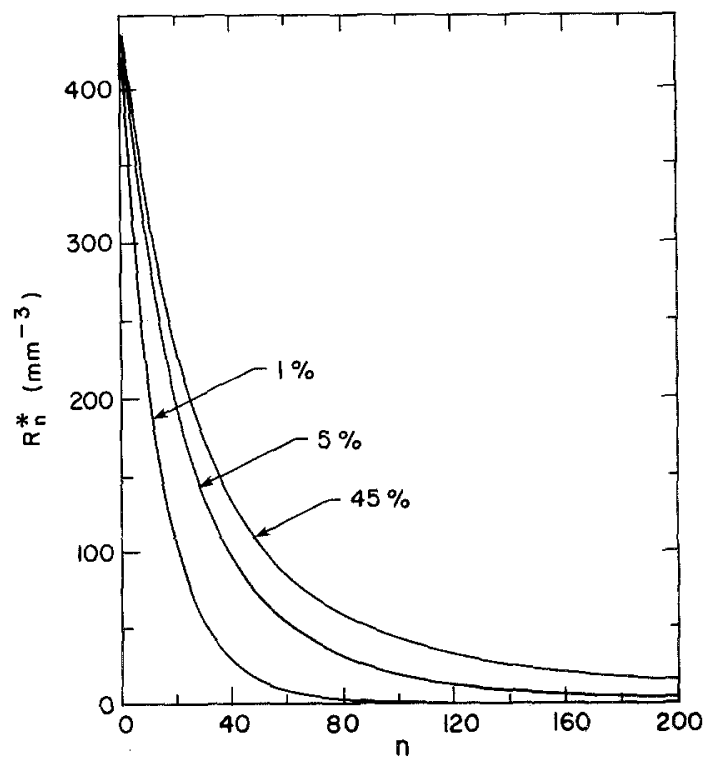

Fig. 6. The number of rooted rouleaux of size $n$ per $\mathrm{mm}^{3}$ of blood plotted against $n$ for the hematocrit, $N / N_{0}$, equal to $1 \%, 5 \%$, and $45 \% . R_{n}^{*}$ has been expressed in concentration units by choosing $N_{0}=10^{7}$ cells $/ \mathrm{mm}^{3} . E_{A}=10 k_{B} T$ and $E_{B}=12 k_{B} T$. 


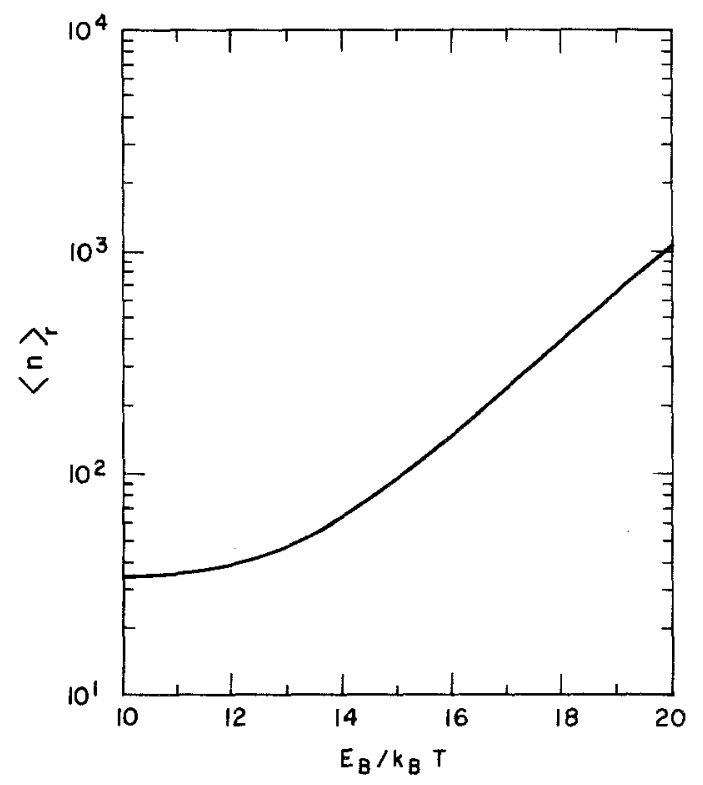

Fig. 7. The mean number of red cells per rooted rouleau, $\langle n\rangle_{r}$, plotted against the branching energy $E_{B}$, with $E_{A}=10 k_{B} T, N_{0}=10^{7}$ cells $/ \mathrm{mm}^{3}$ and $N / N_{0}=5 \%$.

The average rouleau size also increases if either the adhesion energy $E_{A}$ or the branching energy $E_{B}$ increases. In Fig. 7 we show the effect on $\langle n\rangle_{r}$ of increasing $E_{B}$ over the range $E_{B} \leqslant E_{A} \leqslant 2 E_{B}$, with $E_{A}$ held fixed at $10 k_{B} T$. Thus as $E_{B}$ increases, not only are the rouleaux more branched but they are also larger, since each time a branch forms two more cells are added to the rouleau. This qualitative behavior cannot easily be predicted from (4.26) because $\lambda$ changes if $E_{B}$ is changed. However, using the asymptotic results, (4.13) and (4.14), one finds

$$
\langle n\rangle_{r}=\frac{\sum_{n=1}^{\infty} n R_{n}^{*}}{\sum_{n=1}^{\infty} R_{n}^{*}} \cong \frac{\sum_{n=1}^{\infty} n^{-1 / 2} \mu^{n}}{\sum_{n=1}^{\infty} n^{-3 / 2} \mu^{n}} \cong \frac{N}{c N_{0} \phi(\mu, 3 / 2)}
$$

Since $\mu \cong 1$ and $\phi(1,3 / 2) \cong 2.6$,

$$
\langle n\rangle_{r} \cong \frac{N}{2.6 c N_{0}}
$$

Thus the dependence of $\langle n\rangle_{r}$ on $E_{A}$ and $E_{B}$ is through $c$. Combining (7.13) and (4.12) results in

$$
\langle n\rangle_{r} \cong \frac{N}{2.6 N_{0}}\left(\frac{4 \pi \beta^{3 / 2}}{\alpha+2 \sqrt{\beta}}\right)^{1 / 2}
$$

Thus we see $\langle n\rangle_{r}$ increases with $\beta$. If we hold $\alpha$ fixed, then $\langle n\rangle_{r}$ is 
Table IV. Values of $Q_{n}^{r}$ Obtained by Summing

$Q_{n, m}^{r}$ over $m$ and the Approximate Value $Q_{n}^{r}$

Obtained from (4.11) for $E_{A}=10 k_{B} T$

and $E_{B}=12 k_{B} T$

\begin{tabular}{rll}
\hline$n$ & $\left(Q_{n}\right)$ exact & $\left(Q_{n}\right)$ approximate \\
\hline 1 & 2. & $2.4 \times 10^{2}$ \\
2 & $8.8 \times 10^{4}$ & $3.9 \times 10^{6}$ \\
3 & $3.9 \times 10^{9}$ & $9.6 \times 10^{10}$ \\
4 & $1.7 \times 10^{14}$ & $2.9 \times 10^{15}$ \\
5 & $7.5 \times 10^{18}$ & $9.3 \times 10^{19}$ \\
10 & $1.3 \times 10^{42}$ & $6.6 \times 10^{42}$ \\
20 & $3.6 \times 10^{88}$ & $9.2 \times 10^{88}$ \\
40 & $3.3 \times 10^{181}$ & $5.0 \times 10^{181}$ \\
50 & $1.0 \times 10^{228}$ & $1.4 \times 10^{228}$ \\
\hline
\end{tabular}

proportional to $\beta^{1 / 2}$ for $2 \sqrt{\beta} \gg \alpha$ and a semilogarithmic plot of $\ln \langle n\rangle_{r}$ versus $E_{B}$ should be linear with slope $1 / 2$. This linear dependence is exactly what is seen in Fig. 7 as $E_{B}$ becomes large.

To test our asymptotic results more carefully we compare in Table IV the values of $Q_{n}^{r}$ obtained by summing $Q_{n, m}^{r}$ over $m$ with the approximate values of $Q_{n}^{r}$ computed from (4.11). As expected the approximate results are very poor for small $n$, but become increasingly accurate as $n$ increases. Thus for large $n$ we expect the approximate formula (7.5) for the equilibrium rouleau distribution to be accurate. That this is indeed the case is shown in Fig. 8, where we plot for a 5\% hematocrit the exact and asymptotic rouleau distributions. Here we see excellent agreement for $n \geqslant 200$. Because the error in the asymptotic formula for $R_{n}^{*}$ is due only to the error in the asymptotic formula for $Q_{n, m}^{r}$, a quantity whose value is independent of the hematocrit, we obtain precisely the same relative error in the asymptotic formula for $R_{n}^{*}$ at other hematocrits as was obtained in Fig. 8 for $5 \%$ hematocrit.

Our numerical studies of unrooted rouleaux show that our asymptotic formula for $\tilde{R}_{n}^{*},(7.8)$, with its $n^{-5 / 2}$ dependence, is accurate for large $n$. In Fig. 9 we plot for a $5 \%$ hematocrit the values of $\tilde{R}_{n}^{*}$ computed exactly by summing $\tilde{R}_{n, m}^{*}$ as given by (7.2b). Besides the excellent agreement between the exact and asymptotic curves for large values of $n$, one should note that with our standard parameter values of $E_{A}=10 k_{B} T$ and $E_{B}=12 k_{B} T$ large unrooted rouleaux occur with very low frequency. In Figs. 10a and 10b, we show the mean size of, and the mean number of branch points in, an unrooted rouleau computed from (4.24) and (4.28) for different values of the branching energy, $E_{B}$, with $E_{A}=10 k_{B} T$. As $E_{B}$ increases rouleaux become more branched and the possibility of an infinite-sized aggregate becomes more pronounced. At a critical value of $E_{B}$, which we denote $E_{B}^{c}$, 


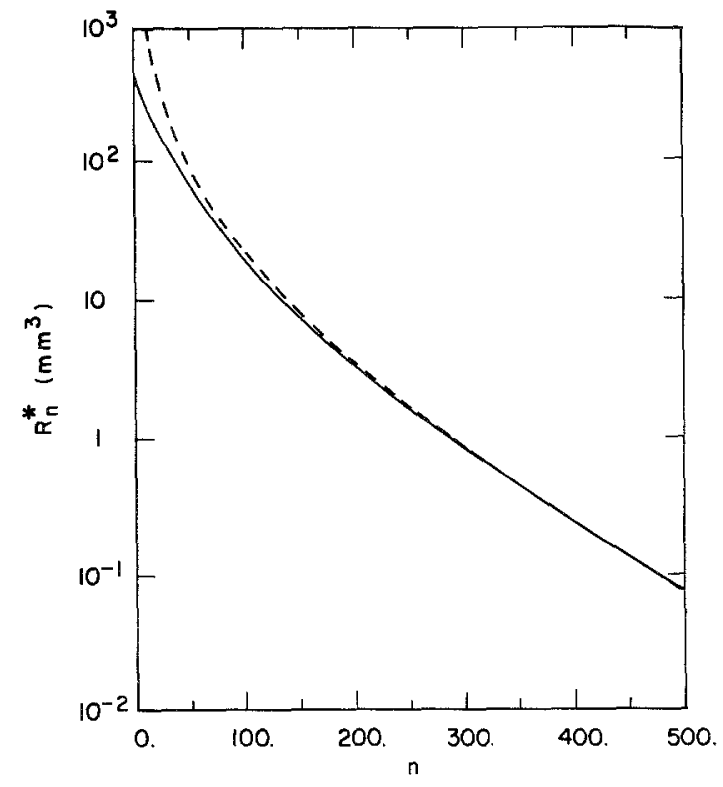

Fig. 8. The number of rooted rouleaux of size $n$ per $\mathrm{mm}^{3}$ of blood computed exactly (solid line) and via the asymptotic formula (7.5) (dotted line) plotted against $n$ for the hematocrit $N / N_{0}=5 \% . E_{A}=10 k_{B} T$ and $E_{B}=12 k_{B} T$.

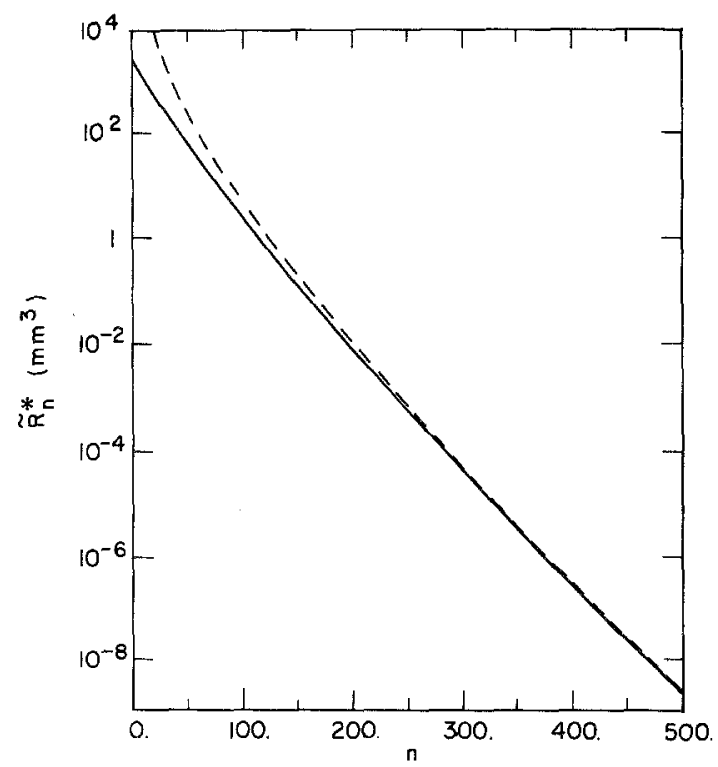

Fig. 9. The number of unrooted rouleaux of size $n$ per $\mathrm{mm}^{3}$ of blood computed exactly (solid line) and via the asymptotic formula (7.8) (dotted line) plotted against $n$ for the hematocrit $N / N_{0}=5 \% . E_{A}=10 k_{B} T$ and $E_{B}=12 k_{B} T$. 

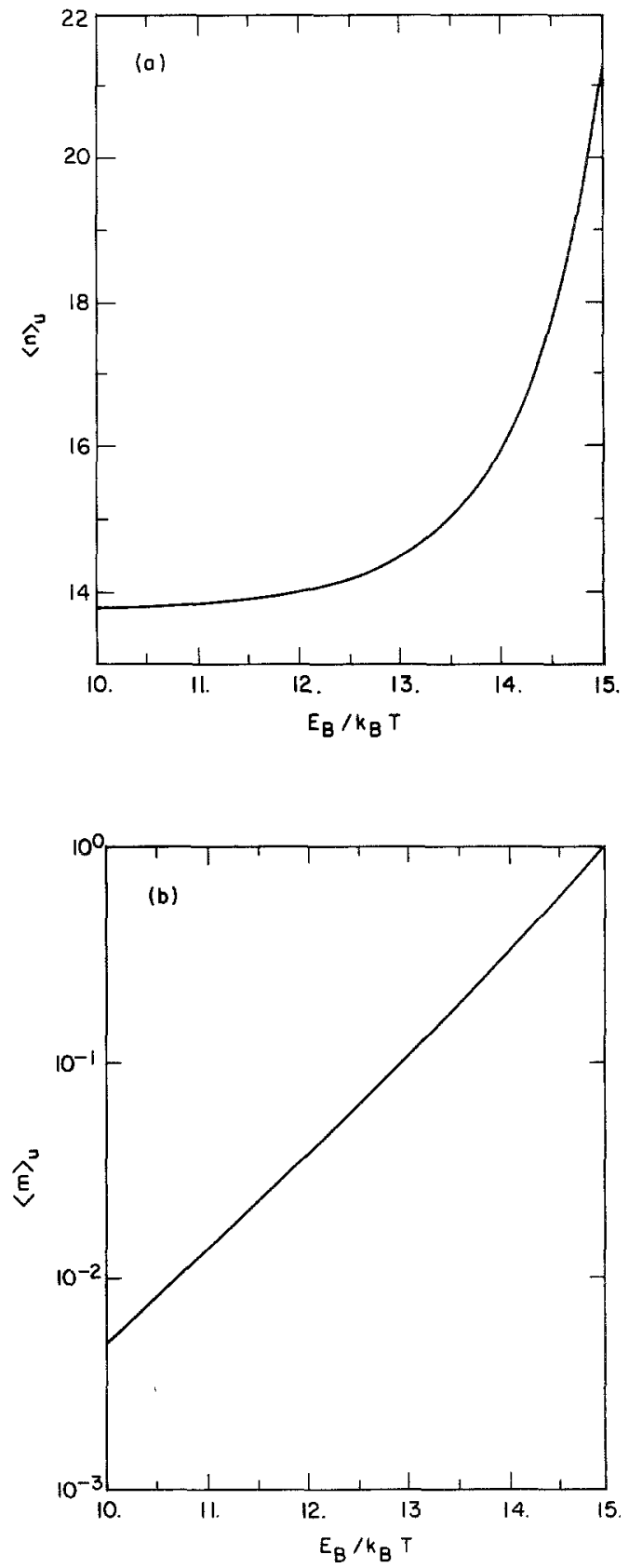

Fig. 10. (a) the mean number of red cells per unrooted rouleau, $\langle n\rangle_{u}$, (b) the mean number of branch points per unrooted rouleau $\langle m\rangle_{u}$, plotted against the branching energy $E_{B}$, with $E_{A}=10 k_{B} T, N_{0}=10^{7}$ cells $/ \mathrm{mm}^{3}$ and $N / N_{0}=5 \%$. 
the condensation condition $U=1$ is met and $\lambda$ can no longer be computed from (7.9). An analytic formula for the critical branching energy $E_{B}^{c}$ can be obtained by setting $U=1$ in (7.9), resulting in

$$
\beta_{c}=\frac{4 \pi N_{0}}{N z}
$$

where $\beta_{c}$ denotes the value of $\beta$ at $U=1$ and $z=\exp (-\lambda)$. Substituting this value of $\beta$ into (7.10) with $U=1$ gives a quadratic equation in $z$ with roots

$$
z=\frac{1}{\alpha}+\frac{8 \pi N_{0}}{\alpha^{2} N}\left[1 \pm\left(1+\frac{\alpha N}{4 \pi N_{0}}\right)^{1 / 2}\right]
$$

Choosing the root which is less than $1 / \alpha$, so that $Q_{0}(z)=z /(1-\alpha z)$ does not go through its singularity, and substituting into (7.15) determines $\beta_{c}$. The critical branching energy $E_{B}^{c}$ is then given by (1.1) and (1.3) as

$$
E_{B}^{c}=k_{B} T \ln \left(\beta_{c} / 4\right)
$$

For the parameters of Fig. $10, E_{B}^{c} \cong 14.98 k_{B} T$.

\section{DISCUSSION}

We have used classical methods of equilibrium statistical mechanics to derive the most probable size distribution and degree of branching of red blood cell aggregates. To count the number of configurations for a rouleau fixed in space, with $n$ cells and $m$ branch points, we represented the rouleau by a rooted tree and used a generating function procedure to count all possible rooted trees. Other methods of graphical enumeration, such as Polya's theorem, could also have been used. ${ }^{(37)}$ The root of the tree corresponded to a face of a red cell which was assumed to be at a fixed location. The remainder of the tree was then "grown" from this root. Because isomorphic rouleaux, that differ only by a rigid rotation, are grown by choosing any end point of a rouleau as the root (see Fig. 4), the number of rooted rouleaux overestimates the actual number of rouleaux. Dividing the number of rooted rouleaux of a given size by the number of end points it contains and accounting for all possible rigid rotations gave the number of (unrooted) rouleaux.

The number of unrooted rouleaux containg $n$ red cells was found to be proportional to $n^{-5 / 2}$ for large $n$. This asymptotic result is similar to the one found by Stockmayer ${ }^{(38)}$ in his study of the random condensation polymerization of $f$-valent molecules. The polycondensation of three-valent particles gives rise to trees identical to the ones studied here. The major difference between our calculation and Stockmayer's is that we allowed 
different energies for chain elongation and branching, whereas Stockmayer assumed that these energies were identical. Methods developed by Gordon $^{(39)}$ and Good ${ }^{(40)}$ based upon branching processes have been used to study polymerization reactions in which branching and elongation occur with different probabilities (cf. Ref. 41). These methods could be, but have not yet been, applied to rouleau formation.

Both our calculation for rouleaux, and calculations for the random polycondensation of $f$-valent molecules with $f \geqslant 3$, show that infinite-sized aggregates can form. In polymer chemistry an infinite-sized aggregate is called a gel. In this paper, we have not attempted to calculate the distribution of sizes of finite rouleaux past the gel point; however, a number of methods of doing so have been proposed in polymer chemistry. ${ }^{(38,40-44)}$ There are indications that rouleaux form which are large enough to be considered a gel. For example, examination of the flow properties of blood at low rates of shear indicates that rouleaux generate three-dimensional structures which impart to blood a yield-stress. ${ }^{(45)}$ The existence of a yield stress means that blood can withstand a finite shearing stress without flowing, a rheological property of a plastic or solid.

A direct comparison of our predicted rouleau size distribution with experimental determinations is difficult for a number of reasons discussed previously. ${ }^{(4)}$ Briefly, we restricted our attention to a highly idealized system in which there was neither fluid flow nor sedimentation of erythrocytes, processes which tend to be of importance in typical experimental setups. Because erythrocytes must collide in order to form rouleaux, equilibrium is established very slowly in systems that rely upon diffusionally driven collisions. Thus, it may be impractical to wait for equilibrium to be established. Nonequilibrium effects can be accounted for by a kinetic theory of rouleau formation developed by Samsel and Perelson. ${ }^{(5,6)}$ Experimentally, the collision process is usually speeded up by randomly mixing the sample ${ }^{(46,47)}$ or by uniformly shearing it. ${ }^{(48-50)}$ Mixing or shearing breaks up rouleaux, ${ }^{(48-50)}$ and thus our theory should predict rouleaux that are larger than are conventionally observed. Lastly, our theory has not taken into consideration finite system effects nor the fact that the number of rouleaux of size $n$ is an integer. As precise experimental determinations of $\tilde{R}_{n, m}^{*}$ become available, refinements to our theory may become justified. Our goal here has been to show how the methods of statistical mechanics can be used to study cellular aggregation phenomena.

\section{ACKNOWLEDGMENTS}

We thank R. W. Samsel for donating the blood and taking the photograph used in Fig. 1. This work was performed under the auspices of 
the U.S. Department of Energy and supported by NATO research grant RG 067.80. A.S.P. is the recipient of an N.I.H. Research Career Development Award K504 AI 00450-03. This work, as the other papers of A.S.P. on rouleau formation, is dedicated to the memory of Aharon KatchalskyKatzir, a great scientist, humanist, and a pioneer of the macromolecular bridging hypothesis.

\section{REFERENCES}

1. R. Fahraeus, Acta Med. Scand. 161:151 (1951).

2. R. R. Reich, Hematology: Physiopathologic Basis for Clinical Practice (Little, Brown and Co., New York, 1978).

3. S. Chien, The Red Blood Cell, Vol. II, D. Surgenor, ed. (Academic Press, New York, 1976), p. 1031.

4. A. S. Perelson and F. W. Wiegel, Biophys. J. 37:515 (1982).

5. R. W. Samsel and A. S. Perelson, Biophys. J. 37:493 (1982).

6. R. W. Samsel and A. S. Perelson, Kinetics of Rouleau Formation. II. Long-Time Behavior, Reversible Reactions and Loop Formation (in preparation).

7. H. L. Goldsmith, J. Gen. Physiol. 52:5s (1968).

8. S. G. Mason and H. L. Goldsmith, Circulatory and Respiratory Mass Transport, G. E. W. Wolstenholme and J. Knight, eds. (Little, Brown \& Co., Boston, 1969), p. 105.

9. F. Gauthier, H. L. Goldsmith, and S. G. Mason, Rheol. Acta 10:344 (1971).

10. F. Gauthier, H. L. Goldsmith, and S. G. Mason, Trans. Soc. Rheol. 15:297 (1971).

11. P. M. Adler, AIChE J. 25:487 (1979).

12. F. W. Wiegel, Lecture Notes in Physics 121 (Springer, Heidelberg, 1980).

13. P. Pfeuty and G. Toulouse, Introduction to the Renormalization Group and to Critical Phenomena (Wiley, London, 1977).

14. P. G. de Gennes, Scaling Concepts in Polymer Physics (Cornell Univ. Press, Ithaca, 1979).

15. D. Stauffer, Physica 106A:177 (1981).

16. R. K. Pathria, Statistical Mechanics (Pergamon, New York, 1972).

17. D. ter Haar, Elements of Statistical Mechanics (Holt, Rinehart and Winston, New York, 1954).

18. K. Huang, Statistical Mechanics (Wiley, New York, 1963).

19. F. Reif, Fundamentals of Statistical and Thermal Physics (McGraw-Hill, New York, 1965).

20. E. Donoghue and J. H. Gibbs, J. Chem. Phys. 70:2346 (1979).

21. E. Donoghue, J. Chem. Phys. 77:4236 (1982).

22. M. Gō, J. Phys. Soc. Jpn. 23:597 (1967).

23. F. W. Wiegel, Conformational Phase Transitions in a Macromolecule: Exactly Solvable Models, in Phase Transitions and Critical Phenomena, Vol. 8, C. Domb and M. S. Green, eds. (Academic, London, 1982).

24. J. Hijmans, J. Chem. Phys. 47:5116 (1967).

25. P. G. de Gennes, Biopolymers 6:715 (1968).

26. F. Harary, Graph Theory (Academic Press, New York, 1969).

27. E. C. Titchmarsh, The Theory of Functions (Oxford Univ. Press, Oxford, 1960).

28. N. G. de Bruijn, Asymptotic Methods in Analysis (North Holland, Amsterdam, 1958).

29. M. Abramowitz and I. A. Stegun, Handbook of Mathematical Functions (Dover, New York, 1970).

30. C. Truesdell, Ann. Math. 46:144 (1945). 
31. H. B. Dwight, Mathematical Tables of Elementary and Some Higher Mathematical Functions (Dover, New York, 1958), pp. 210-215.

32. P. Flory, Principles of Polymer Chemistry (Cornell University Press, Ithaca, 1953).

33. J. B. Miale, Laboratory Medicine Hematology, 5th ed. (Mosby, St. Louis, 1977).

34. S. Chien, In Bioelectrochemistry: Ions, Surfaces, Membranes, M. Blank, ed. (Amer. Chem. Soc., Washington, D.C., 1980), pp. 3-38.

35. S. Chien, In Topics in Bioelectrochemistry and Bioenergetics, Vol. 4, G. Milazzo, ed. (Wiley, New York, 1981), pp. 73-131.

36. T. J. Dekker, Constructive Aspects of the Fundamental Theorem of Algebra, Proc. 1967 Symp. IBM Research Lab. Zürich-Rüschlikon Switzerland, B. Dejon and P. Henrici, eds. (Wiley, New York, 1969), pp. 37-48.

37. F. Harary and E. Palmer, Graphical Enumeration (Academic Press, New York, 1973).

38. W. H. Stockmayer, J. Chem. Phys. 11:45 (1943).

39. M. Gordon, Proc. Roy. Soc. London A268:240 (1962).

40. I. J. Good, Proc. Roy. Soc. London A272:54 (1963).

41. C. A. Macken and A. S. Perelson, An Application of Branching Processes to the Study of Multivalent Ligand-Multivalent Receptor Interactions on Cell Surfaces, Lecture Notes in Biomathematics (Springer Verlag, New York, in press).

42. P. J. Flory, J. Am. Chem. Soc. 63:3091 (1941).

43. G. R. Dobson and M. Gordon, J. Chem. Phys. 41:2389 (1964).

44. R. M. Ziff and G. Stell, J. Chem. Phys. 73:3492 (1980).

45. J. Goldstone, H. Schmid-Schönbein, and R. Wells, Microvasc. Res. 2:273 (1970).

46. E. Ponder, Quart. J. Exp. Physiol. 16:173 (1927).

47. D. Kernick, A. W. L. Jay, S. Rowlands, and L. Skibo, Can. J. Physiol. Pharmacol. 51:690 (1973).

48. H. Schmid-Schönbein, P. Gaehtgens, and H. Hirsh, J. Clin. Invest. 47:1447 (1968).

49. H. Schmid-Schönbein, E. Volger, and H. J. Klose, Pflïgers Arch. 333:140 (1972).

50. S. Usami, R. G. King, S. Chien, R. Skalak, C. R. Huang, and A. L. Copley, Biorheology 12:323 (1975). 